\title{
The Power of Salinity Gradients: An Australian Example
}

Fernanda Helfer ${ }^{1}$ and Charles Lemckert ${ }^{2}$

${ }^{1,2}$ School ofEngineering, Griffith University, Australia

${ }^{1}$ Corresponding author.Email:f.helfer@griffith.edu.au,Phone: +61 0755527378.

Postal address: Griffith School of Engineering(G09), Southport, QLD 4222, Australia.

Abstract: The development and exploitation of sustainable and environmentally friendly energy sources are required in order to resolve global energy shortages and to reduce the reliance of many countries on fossil fuel combustion. Salinity gradient energy has been considered a potential candidate for renewable energy due to the abundance of saline waters that could be combined with less saline solutions across the globe. Pressure Retarded Osmosis (PRO) is one of the technologies to harness salinity gradient energy. Apart from zero carbon dioxide emission, PRO is capable of producing power with less periodicity, abundance and low environmental impacts. One of the pre-conditions for the technical and financial feasibility of PRO, however, is the development of a PRO-specific membrane - one that meets the conditions that none of the current commercially-available membranes have met so far. The current paper discusses the progress made in PRO membrane development, particularly during the past decade, and analyses the challenges that are still hindering the implementation of PRO at large scales. Also, this paper explores possibilities for the implementation of PRO by analysing various combinations of existent solutions of various salt concentrations. Australia has been chosen to demonstrate some potential applications of PRO. This vast country has extensive reserves of saline waters that could be paired with less concentrated solutions to generate power. For each combination of solutions, a conceptual idea is presented, and an estimate of power production is given. Also, advantages and disadvantages of each scheme are discussed. The ideas and estimates can be easily extrapolated, with minor adjustments, to other countries with similar conditions. It is hoped that this publication will be valuable to those nations that have similar policies as Australia's, with government incentives for the development and implementation of new technologies to explore new renewable energy sources.

Keywords: salinity, osmotic pressure, pressure retarded osmosis, renewable energy 


\section{Introduction}

Salinity gradient energy is the energy released upon the mixing of waters with different salt concentrations. This energy is released, for example, when rivers meet the ocean. About 0.70 - $0.75 \mathrm{kWh}(2.5$ - $2.7 \mathrm{MJ})$ is dissipated when $1 \mathrm{~m}^{3}$ of river water mixes with $1 \mathrm{~m}^{3}$ of sea water [1]. Pressure Retarded Osmosis (PRO), which relies on osmotic driven flow through a membrane separating fresher water from saltier water, is one of the mechanisms able to harness the energy of salinity gradients. The power generated through PRO is usually referred to as "osmotic power". With PRO, if one tenth of the global river discharge (i.e., approximately $3,700 \mathrm{~km} \mathrm{y}^{-1}$ ) was used for power production from the mixing with sea water, more than 1,300 TWh per year (0.16 TW of instant power) could be generated. This is equivalent to half the annual power generation in the European Union [2], and equal to the global potential production of hydroelectric power [3]. Table 1 shows the potential for osmotic power production of some of the major sources of fresh water in the world if these were mixed with sea water.

Table 1. Potential for osmotic power production from some major world rivers. Reprinted from Ref. [4], copyright (2014), with permission from Elsevier.

\begin{tabular}{lccc}
\hline \multicolumn{1}{c}{ Source of fresh water } & $\begin{array}{c}\text { Average flow rate } \\
\left(\mathrm{m}^{3} \mathrm{~s}^{-1}\right)\end{array}$ & Power (MW) $^{2}$ & $\begin{array}{c}\text { Electricity supply } \\
\text { (thousands of households) }^{1}\end{array}$ \\
\hline World & $1.2 \times 10^{6}$ & 124,800 & N/A \\
Amazon River, Brazil & $2 \times 10^{\mathrm{b}}$ & 20,800 & 77,600 \\
La Plata - Parana River, Argentina & $8 \times 10^{4}$ & 8,320 & 29,100 \\
Congo River, Congo Angola & $5.7 \times 10^{4}$ & 5,930 & 282,300 \\
USA & $5.4 \times 10^{4}$ & 5,620 & 4,000 \\
Yangtze River, China & $2.2 \times 10^{4}$ & 2,290 & 5,800 \\
Ganges River, Bangladesh & $2 \times 10^{4}$ & 2,080 & 74,300 \\
Mississippi River, USA & $1.8 \times 10^{4}$ & 1,870 & 7,300 \\
Columbia River, USA & $7.5 \times 10^{3}$ & 780 & 550 \\
\hline
\end{tabular}

${ }^{1}$ Based on the average household consumption per country reported in Ref. [5]

${ }^{2}$ The power was estimated by assuming $10 \%$ of each river's average discharge, and a power output of $1 \mathrm{MW}^{\mathrm{M}}$ per $\mathrm{m}^{3} \mathrm{~s}^{-1}$ of river water (i.e., $40 \%$ energy conversion efficiency, as suggested in the literature [6]).

PRO research and development is still in its infancy. Although the mechanism was discovered in 1973, it was not until 2009 that it began to be seen as a promising source of renewable energy. This was due to the rapid advances in membrane technology and the decline in membrane prices observed in the late 2000s as a result of the growth of the desalination and water treatment industries. Although membrane technology has evolved significantly over time, the feasibility of PRO, both technical and economical, are still predicated, to a great extent, on the development of a PRO-specific membrane that meets some pre-determined 
criteria. Among these criteria, are the high water permeability of the membrane, the high salt rejection rate, the physical resistance of the membrane to high applied pressures and the low susceptibility of the membrane to concentration polarization (i.e., salt built-up at the membrane's interfaces). Once the right membrane technology becomes available in the market, PRO is believed to become competitive with the other conventional sources of renewable energy, such as wind and solar.

Within this context, one of the objectives of this article is to discuss the latest developments in PRO, with a particular emphasis on the progress made in the development of new membranes for this particular purpose. This study is, in part, an update of our previous publication on PRO, in which we also discussed the progress made regarding membrane development [4]. As significant progress in membrane development has been made since that publication, new findings have been compiled and discussed in this new publication to assist in the development of further research in the PRO field. Another objective of this study is to analyse some possibilities for the implementation of PRO in large scale, by looking into different sources of natural solutions that could be combined to generate osmotic power. Potential locations and conditions under which PRO could be implemented are indicated, using Australia's water resources as examples. For each example, a conceptual idea and an estimate of the power production are provided. Although the examples given in this article focus on Australia, the same favourable conditions that make salinity gradient a potential source of renewable energy for this country can also be encountered in many other countries, making the conceptual ideas presented here warrant worldwide consideration.

\section{Power Production with Pressure Retarded Osmosis - Review of Concepts}

The term 'osmosis' refers to the transport of pure water across a semipermeable membrane from a solution of lower osmotic pressure (lower salt concentration), called 'feed solution', to a solution of higher osmotic pressure (higher salt concentration), called 'draw solution'. The pure water flows through this membrane to the draw solution side in order to equalise the salt concentrations on the two sides. The semipermeable membrane is designed to allow the passage of water, rejecting the passage of solute molecules or ions. Every solution is characterised by an intrinsic 'osmotic pressure', which is determined by the amount of salt in the solution and calculated according to the van't Hoff equation (refer to [4] for formulations). In simple terms, the osmotic pressure of a solution represents the pressure which, when applied 
to the solution, would stop the flux of pure water across the semipermeable membrane, required to equalise the salt concentration. For sea water for example, in which the $\mathrm{NaCl}$ concentration varies between $3.0 \%$ and $4.0 \%$ (or approximately $30-40 \mathrm{~g} \mathrm{~L}^{-1}$ ), the osmotic pressure falls between 25 and 33 bar, if the temperature is around $25^{\circ} \mathrm{C}$. This means that a pressure equivalent to 25 - 33 bar should be applied to the sea water in order to prevent the flow of pure water, should these two solutions be separated by a semipermeable membrane. Solutions with high osmotic pressures are, for instance, concentrated brine remaining from desalination plants, and brine formed within salt lakes, such as the Great Salt Lake (USA), the Aral Sea (Kazakhstan and Uzbekistan), the Dead Sea (Israel) and Lake Eyre (Australia). The salinity of concentrated brine from desalination plants varies between $6 \%$ and $7 \%$, which translates into an osmotic pressure varying between 50 and 59 bar. The salinity of salt lakes could be as high as 34\% (Dead Sea, for example), meaning an osmotic pressure of 290 bar. For fresh water, the osmotic pressure is close to zero as a result of its negligible amount of salts.

When two solutions are separated by a semipermeable membrane, the theoretical maximum flux through the membrane is directly proportional to the permeability of the membrane and to the difference in pressure between the two solutions [7]:

$$
J=A(\Delta \pi-\Delta P)
$$

where $J$ is the water flux through the membrane (typically in $\mathrm{L} \mathrm{m}^{-2} \mathrm{~h}^{-1}$ ); $\Delta \pi=\pi_{D}-\pi_{F}$, where $\Delta \pi$ is the difference in osmotic pressure across the membrane, $\pi_{D}$ is the osmotic pressure in the draw solution (more concentrated solution) and $\pi_{F}$ is the osmotic pressure in the feed solution (less concentrated solution), both in bar; $\Delta P=P_{D}-P_{F}$ is the difference in hydrostatic pressure between the two solutions; $P_{D}$ is the pressure in the draw solution and $P_{F}$ is the pressure in the feed soution, also in bar; and $A$ is the intrinsic water permeability coefficient of the membrane, a parameter that depends on the properties of the membrane in $\mathrm{L} \mathrm{m}^{-2} \mathrm{~h}^{-1} \mathrm{bar}^{-1}$.

In Forward Osmosis (FO) or simply 'osmosis', the flux is solely driven by the osmotic pressure difference, that is, $J=A \Delta \pi$. When the applied pressure differential, $\Delta P$, is increased by for example pressurizing the draw solution side, and has a magnitude between 0 and $\Delta \pi$, the water flux through the membrane is still predominantly driven by the osmotic pressure difference $(\Delta \pi)$, but the flow is declined due to the increasing $\Delta P$. This osmotic process is called Pressure 
Retarded Osmosis (PRO). It is on PRO that the production of osmotic power is based. For a steady production of power, the draw solution has to be maintained under constant hydrostatic pressure so as to provide a steady flow of pure water through the membrane, increasing the volume on the side of the draw solution. This additional volume can then be used to generate power.

PRO was suggested as a mechanism to harness salinity gradient energy in 1973 in Israel [810], but the high membrane prices and the infant membrane technology at that time restrained the large-scale implementation of PRO power plants. The continuing growth of the desalination and water treatment industries brought about higher performing membranes, reviving research on PRO in the late 2000s.

In 2009, the Norwegian state-owned company Statkraft opened the first prototype of an osmotic power plant working on the principles of PRO. The Norwegian prototype has been proving the feasibility of osmotic power generation using sea water and fresh water as draw and feed solutions, respectively. The plant is being used in the testing of different types of membranes and plant configurations. A general sketch of the Statkraft's power plant is shown in Figure 1. The plant is equipped with $2000 \mathrm{~m}^{2}$ of membranes and has a marginal power output between $2 \mathrm{~kW}$ and $4 \mathrm{~kW}$.

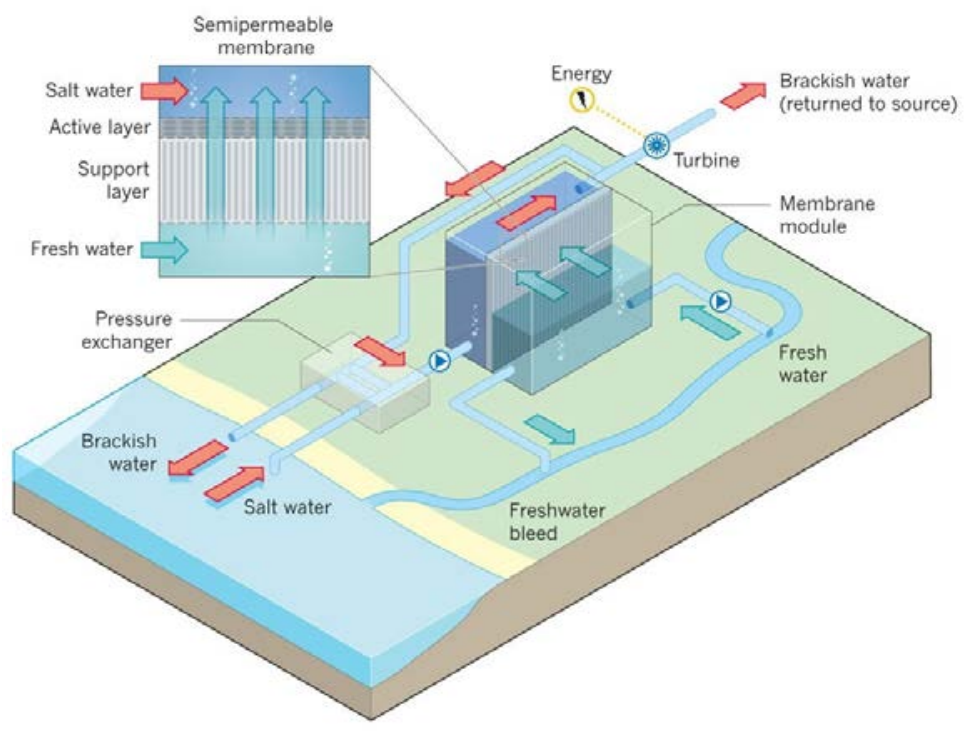

Figure 1. A typical simplified PRO process used in the Statkraft's osmotic power plant prototype. Reprinted by permission from Macmillan Publishers Ltd: Nature [11], copyright (2012). 
Figure 2 depicts an idealized osmotic power production system with continuous, steady state flow. First, a more concentrated solution of volume $V$ and with osmotic pressure $\pi_{D}$ is pumped into the plant at a hydraulic pressure $P_{D}$. The power input is given by the product of the volume flow $(V)$ and the applied pressure $P_{D}$. At the same time, a less concentrated solution enters the permeator on the opposite side of the membrane at osmotic and hydraulic pressures that are very low in comparison to these quantities on the concentrated side. Pure water permeates the membrane from the less concentrated solution side to the more concentrated solution side at a rate $\Delta V$ (note $\Delta V=J A_{m}$, where $A_{m}$ is the membrane area) and acquires a pressure $P_{D}$. Therefore, the flow in the more concentrated solution side is augmented by $\Delta V$. The new flow $(V+\Delta V)$ enters a hydroturbine in which $P_{D}$ is reduced to zero, as it delivers power of magnitude $P_{D}(V$ $+\Delta V)$.

It can be seen from Figure 2 that the maximum net power ( $W_{N E T}^{M A X}$ ) that could be possibly produced in this ideal scheme is the difference between the quantity delivered (i.e., $P_{D}(V+$ $\Delta V)$ ) and the power added to the system (i.e., $P_{D} V$ ), as shown below:

$W_{N E T}^{M A X}=P_{D}(V+\Delta V)-P_{D} V=P_{D} \Delta V$

It should be noted that this condition is for an efficiency of $100 \%$. For mechanical efficiencies less than $100 \%$, the net power would be $P_{D} \Delta V \eta$ where $\eta$ is the mechanical efficiency. For example, the osmotic power plant prototype in Norway has been able to generate 1.0 MJ per 1 $\mathrm{m}^{3}$ of fresh water (or 1.0 MW of power per $1 \mathrm{~m}^{3} \mathrm{~s}^{-1}$ of fresh water). The maximum energy released when $1 \mathrm{~m}^{3}$ of fresh water mixes with the ocean is around 2.6 MJ, meaning the efficiency of the Statkraft's power plant is around 40\% [6]. 


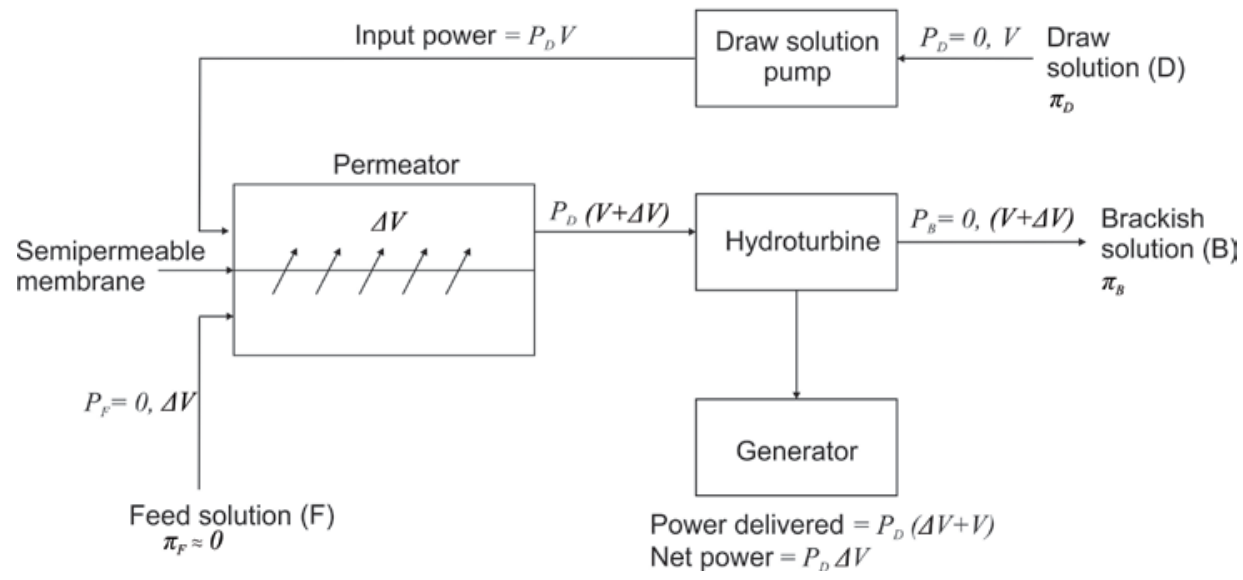

Figure 2. Schematic representation of an idealized PRO system. This system assumes $100 \%$ efficiency of rotating components, inexistence of friction losses in the plant streams and membranes perfectly semipermeable. Reprinted from Ref [4], copyright (2014), with permission from Elsevier.

Membrane power density (power production per unit area of membrane) is an important factor in osmotic power, since it determines the amount of membrane required in the plant. The higher the membrane power density, the less the membrane area required, and the less the associated costs with plant installation and maintenance. For a large scale osmotic power plant operating using river water and sea water, the minimum power density required for the financial viability of the entire system is $5 \mathrm{~W} \mathrm{~m}^{-2}[2,12,13]$. The membrane power density (i.e., the power production per unit area of membrane) $(D)$ - is calculated as:

$$
D=J P_{D}
$$

By replacing $J$ in Eq. 3 with $A(\Delta \pi-\Delta P)$, and $\Delta P$ by $P_{D}$ (assuming $P_{F}$ is equal or close to zero), and differentiating the resulting expression with respect to $P_{D}$, it can be seen that $D$ reaches a maximum when the operating pressure is half the osmotic pressure difference between the two solutions (i.e., $P_{D}=\Delta \pi / 2$ ). 


\section{Actual Membrane Performances}

Table 2 shows power densities obtained under laboratory conditions for various types of membrane using sea water as the draw solution. It can be clearly seen the advancements made in membrane development over the years. The initial studies on PRO utilised membranes designed for RO and FO, from which only minor power densities could be achieved. In the last years, with research turned towards the development of membranes made specifically for PRO, power densities up to $11.5 \mathrm{~W} \mathrm{~m}^{-2}$ have been reported in the literature.

Table 2. Summary of experimental results using combinations of solutions representing either fresh water vs. sea water or brackish water vs. sea water PRO schemes. Updated from Ref. [4]

\begin{tabular}{|c|c|c|c|c|c|c|}
\hline $\begin{array}{l}\text { Feed } \\
\text { solution }\end{array}$ & $\begin{array}{l}\text { Water flux } \\
\left(\mathrm{L} \mathrm{m}^{-2} \mathrm{~h}^{-1}\right)\end{array}$ & $\begin{array}{l}\text { Operating } \\
\text { pressure } \\
\text { (bar) }\end{array}$ & $\begin{array}{l}\text { Power density } \\
\qquad\left(\mathrm{W} \mathrm{m}^{-2}\right)\end{array}$ & Membrane type & Year & Source \\
\hline $\begin{array}{c}\text { Fresh water } \\
(<0.05 \% \mathrm{NaCl})\end{array}$ & 1.0 & 12.0 & 0.4 & RO Permasep B-10 hollow fiber membrane & 1976 & $\begin{array}{l}\text { Loeb et al. } \\
{[10]}\end{array}$ \\
\hline $\begin{array}{l}\text { Fresh water } \\
(<0.05 \% \mathrm{NaCl})\end{array}$ & N/A & N/A & 1.3 & Modified cellulose acetate membrane & 2008 & $\begin{array}{l}\text { Gerstandt et } \\
\text { al. [14] }\end{array}$ \\
\hline $\begin{array}{c}\text { Fresh water } \\
(<0.05 \% \mathrm{NaCl})\end{array}$ & N/A & N/A & 3.5 & Modified thin-film composite membrane & 2008 & $\begin{array}{l}\text { Gerstandt et } \\
\text { al. [14] }\end{array}$ \\
\hline $\begin{array}{c}\text { Fresh water } \\
(<0.05 \% \mathrm{NaCl})\end{array}$ & 8.2 & 7.0 & 1.6 & $\begin{array}{l}\text { Commercial cellulose acetate membrane } \\
\text { from Osmonics }\end{array}$ & 2009 & $\begin{array}{l}\text { Thorsen and } \\
\text { Holt [6] }\end{array}$ \\
\hline $\begin{array}{l}\text { Brackish water } \\
(\approx 0.5 \% \mathrm{NaCl})\end{array}$ & 8.2 & 9.6 & 2.2 & $\begin{array}{l}\text { Commercial flat sheet cellulose triacetate } \\
\text { FO membrane from HTI }\end{array}$ & 2009 & $\begin{array}{c}\text { Achilli et al. } \\
\text { [15] }\end{array}$ \\
\hline $\begin{array}{l}\text { Brackish water } \\
(\approx 0.25 \% \mathrm{NaCl})\end{array}$ & 9.0 & 9.6 & 2.4 & $\begin{array}{l}\text { Commercial flat sheet cellulose triacetate } \\
\text { FO membrane from HT I }\end{array}$ & 2009 & $\begin{array}{c}\text { Achilli et al. } \\
{[15]}\end{array}$ \\
\hline $\begin{array}{c}\text { DI water } \\
(0.0 \% \mathrm{NaCl})\end{array}$ & 10.1 & 9.7 & 2.7 & $\begin{array}{l}\text { Commercial flat sheet cellulose triacetate } \\
\text { FO membrane from HT I }\end{array}$ & 2009 & $\begin{array}{l}\text { Achilli et al. } \\
{[15]}\end{array}$ \\
\hline $\begin{array}{c}\text { Fresh water } \\
(<0.05 \% \mathrm{NaCl})\end{array}$ & 8.1 & 11.9 & 2.7 & TFC membrane from GKSS, Germany & 2009 & $\begin{array}{l}\text { Thorsen and } \\
\text { Holt [6] }\end{array}$ \\
\hline $\begin{array}{c}\text { Fresh water } \\
(<0.05 \% \mathrm{NaCl})\end{array}$ & 5.0 & 8.8 & 1.2 & $\begin{array}{l}\text { Commercial FO asymmetric cellulose } \\
\text { acetate membrane supported by a woven } \\
\text { fabric from HTI }\end{array}$ & 2012 & $\begin{array}{l}\text { She et al. } \\
{[16]}\end{array}$ \\
\hline $\begin{array}{c}\text { Fresh water } \\
(<0.05 \% \mathrm{NaCl})\end{array}$ & 10.0 & 8.1 & 2.3 & $\begin{array}{c}\text { Improved flat sheet cellulose triacetate } \\
\text { membrane from HTI }\end{array}$ & 2012 & $\begin{array}{l}\text { Schiestel et } \\
\text { al. [17] }\end{array}$ \\
\hline $\begin{array}{l}\text { Waste water } \\
(\approx 0.5 \% \mathrm{NaCl})\end{array}$ & 16.7 & 8.9 & 4.1 & $\begin{array}{l}\text { Cust omized TFC hollow fiber membrane } \\
\text { for PRO }\end{array}$ & 2012 & $\begin{array}{l}\text { Chou et al. } \\
\text { [18] }\end{array}$ \\
\hline $\begin{array}{l}\text { Waste water } \\
(\approx 0.2 \% \mathrm{NaCl})\end{array}$ & 22.7 & 8.9 & 5.6 & $\begin{array}{l}\text { Customized TFC hollow fiber membrane } \\
\text { for PRO }\end{array}$ & 2012 & $\begin{array}{l}\text { Chou et al. } \\
\text { [18] }\end{array}$ \\
\hline $\begin{array}{l}\text { River water } \\
(<0.05 \% \mathrm{NaCl})\end{array}$ & 32.0 & 6.4 & 5.7 & $\begin{array}{l}\text { Customized TFC hollow fiber membrane } \\
\text { for PRO }\end{array}$ & 2012 & $\begin{array}{l}\text { Chou et al. } \\
{[18]}\end{array}$ \\
\hline $\begin{array}{c}\text { DI water } \\
(0.0 \% \mathrm{NaCl})\end{array}$ & 25.0 & 13.0 & 9.0 & $\begin{array}{l}\text { Pre-treated TFC flat sheet membrane with } \\
\text { modified support layer }\end{array}$ & 2013 & $\begin{array}{l}\text { Han et al. } \\
\text { [19] }\end{array}$ \\
\hline $\begin{array}{l}\text { DI water } \\
(0.0 \% \mathrm{NaCl})\end{array}$ & 20.7 & 13.0 & 7.5 & $\begin{array}{l}\text { TFC membrane with an embedded woven } \\
\text { mesh supported by tricot fabric feed } \\
\text { spacers }\end{array}$ & 2013 & $\begin{array}{l}\text { Straub et al. } \\
\quad[20]\end{array}$ \\
\hline $\begin{array}{l}\text { DI water } \\
(0.0 \% \mathrm{NaCl}\end{array}$ & 27.5 & 15.0 & 11.5 & $\begin{array}{l}\text { TFC polyethersulfone, hollow fiber } \\
\text { membrane with a sponge-like porous } \\
\text { structure }\end{array}$ & 2014 & $\begin{array}{l}\text { Zhang et al. } \\
\text { [21] }\end{array}$ \\
\hline
\end{tabular}

Table 3, in turn, shows the power densities obtained under laboratory conditions for various membranes using concentrated brine $(\mathrm{NaCl}$ concentration $>6 \%)$ as the draw solution. A 
maximum power density of $60 \mathrm{~W} \mathrm{~m}^{-2}$ has been reported in the literature for a thin-film composite membrane re-designed to stand high applied pressures under PRO conditions [20]. This high power density was measured using a highly concentrated draw solution $\approx \approx 18 \%$ $\mathrm{NaCl}$ ) and pure water as feed solution. For solutions simulating salt concentrations of desalination brine $\left(\approx 6 \% \mathrm{NaCl}\right.$ concentration), power densities as high as $24.3 \mathrm{~W} \mathrm{~m}^{-2}$ have been reached under laboratory conditions.

It is important to note that the water fluxes, and hence power densities, observed under laboratory conditions (second column in Table 2 and third column in Table 3) are actually much lower than the maximum theoretical fluxes obtained from Eq 1, in which the flux is calculated as a function of the intrinsic permeability of the membranes multiplied by the osmotic pressure difference between the solutions minus the applied pressure on the draw solution side. The reason for this difference is attributed to concentration polarization - the reduced effective osmotic pressure difference between the two solutions due to salt built-up at the membrane interfaces. Since ideal semipermeable membranes with $100 \% \mathrm{NaCl}$ rejection do not exist, a small quantity of salt leaks to the freshwater side of the membrane. As a result, the salt concentration near the membrane rises and the osmotic pressure difference drops. For example, considering a PRO scheme using a highly concentrated draw solution (e.g., 18\% $\mathrm{NaCl}$ ) and pure water as feed solution, operating at an applied pressure of 48 bar, and using a membrane with a water permeability coefficient of $2.49 \mathrm{~L} \mathrm{~m}^{-2} \mathrm{~h}^{-1} \mathrm{bar}^{-1}$ [20], the theoretical flux would result in $255 \mathrm{~L} \mathrm{~m}^{-2} \mathrm{~h}^{-1}$ and the corresponding power density, in $340 \mathrm{~W} \mathrm{~m}^{-2}$. This same scheme tested under laboratory conditions has actually yielded a membrane flux of $45 \mathrm{~L} \mathrm{~m}^{-2} \mathrm{~h}^{-}$ 1 and a power density of $60 \mathrm{~W} \mathrm{~m}^{-2}$ [20]. Nevertheless, the concentration polarization issue is gradually being reduced with research and development of new membranes (e.g., [20-22]). 
Table 3. Summary of experimental results using concentrated brine $(\mathrm{NaCl}$ concentration $>6 \%)$ as draw solution and fresh or brackish water as feed solution. Updated from Ref. [4]

\begin{tabular}{|c|c|c|c|c|c|c|c|}
\hline $\begin{array}{l}\text { Feed } \\
\text { solution }\end{array}$ & $\begin{array}{l}\text { Draw } \\
\text { solution }\end{array}$ & $\begin{array}{l}\text { Water flux } \\
\left(\mathrm{L} \mathrm{m}^{-2} \mathrm{~h}^{-1}\right)\end{array}$ & $\begin{array}{l}\text { Operating } \\
\text { pressure } \\
\text { (bar) }\end{array}$ & $\begin{array}{l}\text { Power } \\
\text { density } \\
\left(\mathrm{W} \mathrm{m} \mathrm{m}^{-2}\right)\end{array}$ & Membrane type & Year & Source \\
\hline $\begin{array}{l}\text { Brackish water } \\
(\approx 0.2 \% \mathrm{NaCl})\end{array}$ & $\begin{array}{l}\text { Brine }(6 \% \\
\quad \mathrm{NaCl})\end{array}$ & 16.2 & 8.9 & 4.0 & $\begin{array}{l}\text { Flat sheet cellulose triacetate } \\
\text { membrane }\end{array}$ & 2009 & $\begin{array}{l}\text { Achilli } \\
\text { et al. } \\
{[15]}\end{array}$ \\
\hline $\begin{array}{l}\text { Brackish water } \\
(\approx 0.5 \% \mathrm{NaCl})\end{array}$ & $\begin{array}{l}\text { Brine }(6 \% \\
\quad \mathrm{NaCl})\end{array}$ & 16.2 & 8.9 & 4.0 & $\begin{array}{l}\text { Flat sheet cellulose triacetate } \\
\text { membrane }\end{array}$ & 2009 & $\begin{array}{l}\text { Achilli } \\
\text { et al. } \\
\text { [15] }\end{array}$ \\
\hline $\begin{array}{c}\text { DI water } \\
(0.0 \% \mathrm{NaCl})\end{array}$ & $\begin{array}{l}\text { Brine }(6 \% \\
\quad \mathrm{NaCl})\end{array}$ & 18.8 & 9.7 & 5.1 & $\begin{array}{l}\text { Commercial flat sheet cellulose } \\
\text { triacetate FO membrane from HTI }\end{array}$ & 2009 & $\begin{array}{l}\text { Achilili } \\
\text { et al. } \\
{[15]}\end{array}$ \\
\hline $\begin{array}{l}\text { Waste water } \\
(\approx 0.5 \% \mathrm{NaCl})\end{array}$ & $\begin{array}{l}\text { Brine }(6 \% \\
\quad \mathrm{NaCl})\end{array}$ & 11.0 & 11.8 & 3.6 & $\begin{array}{l}\text { Commercial FO asymmetric cellulose } \\
\text { acetate membrane from HT I }\end{array}$ & 2012 & $\begin{array}{l}\text { She et } \\
\text { al. } \\
{[16]}\end{array}$ \\
\hline $\begin{array}{c}\text { River water } \\
(<0.05 \% \mathrm{NaCl})\end{array}$ & $\begin{array}{l}\text { Brine }(6 \% \\
\mathrm{NaCl})\end{array}$ & 11.8 & 11.6 & 3.8 & $\begin{array}{l}\text { Commercial FO asymmetric cellulose } \\
\text { acetate membrane from HT I }\end{array}$ & 2012 & $\begin{array}{l}\text { She et } \\
\text { al. } \\
{[16]}\end{array}$ \\
\hline $\begin{array}{l}\text { Waste water } \\
(\approx 0.5 \% \mathrm{NaCl})\end{array}$ & $\begin{array}{l}\text { Brine }(6 \% \\
\quad \mathrm{NaCl})\end{array}$ & 14.0 & 11.5 & 4.5 & $\begin{array}{l}\text { Commercial FO asymmetric cellulose } \\
\text { acetate membrane from HTI, } \\
\text { supported by a woven fabric }\end{array}$ & 2012 & $\begin{array}{l}\text { She et } \\
\text { al. } \\
{[16]}\end{array}$ \\
\hline $\begin{array}{l}\text { Waste water } \\
(\approx 0.5 \% \mathrm{NaCl})\end{array}$ & $\begin{array}{l}\text { Brine } \\
(12 \% \\
\mathrm{NaCl})\end{array}$ & 19.0 & 12.6 & 6.7 & $\begin{array}{l}\text { Commercial FO asymmetric cellulose } \\
\text { acetate membrane from HTI, } \\
\text { supported by a woven fabric }\end{array}$ & 2012 & $\begin{array}{l}\text { She et } \\
\text { al. } \\
{[16]}\end{array}$ \\
\hline $\begin{array}{l}\text { Treated waste water } \\
\quad(<0.05 \% \mathrm{NaCl})\end{array}$ & $\begin{array}{l}\text { Brine }(6 \% \\
\mathrm{NaCl})\end{array}$ & 11.0 & 25.1 & 7.7 & $\begin{array}{l}\text { Hollow fibre modules from Toyobo } \\
\text { Co. Ltd. }\end{array}$ & 2012 & $\begin{array}{l}\text { Saito } \\
\text { et al. } \\
{[23]}\end{array}$ \\
\hline $\begin{array}{l}\text { Waste water } \\
(\approx 0.5 \% \mathrm{NaCl})\end{array}$ & $\begin{array}{l}\text { Brine }(6 \% \\
\mathrm{NaCl})\end{array}$ & 33.3 & 9.1 & 8.4 & $\begin{array}{l}\text { Cust omized TFC hollow fiber } \\
\text { membrane for PRO }\end{array}$ & 2012 & $\begin{array}{l}\text { Chou } \\
\text { et al. } \\
{[18]}\end{array}$ \\
\hline $\begin{array}{l}\text { Waste water } \\
(\approx 0.2 \% \mathrm{NaCl})\end{array}$ & $\begin{array}{l}\text { Brine }(6 \% \\
\mathrm{NaCl})\end{array}$ & 42.5 & 9.0 & 10.6 & $\begin{array}{l}\text { Customized TFC hollow fiber } \\
\text { membrane for PRO }\end{array}$ & 2012 & $\begin{array}{l}\text { Chou } \\
\text { et al. } \\
{[18]}\end{array}$ \\
\hline $\begin{array}{c}\text { River water } \\
(<0.05 \% \mathrm{NaCl})\end{array}$ & $\begin{array}{l}\text { Brine }(6 \% \\
\mathrm{NaCl})\end{array}$ & 47.2 & 8.4 & 11.0 & $\begin{array}{l}\text { Cust omized TFC hollow fiber } \\
\text { membrane for PRO }\end{array}$ & 2012 & $\begin{array}{l}\text { Chou } \\
\text { et al. } \\
{[18]}\end{array}$ \\
\hline $\begin{array}{l}\text { Brackish water } \\
(\approx 0.5 \% \mathrm{NaCl})\end{array}$ & $\begin{array}{l}\text { Brine }(6 \% \\
\quad \mathrm{NaCl})\end{array}$ & 18.3 & 15.2 & 7.7 & $\begin{array}{l}\text { Pre-treated flat sheet TFC membrane } \\
\text { based on nanofiber substrate }\end{array}$ & 2013 & $\begin{array}{l}\text { Song } \\
\text { et al. } \\
{[22]}\end{array}$ \\
\hline $\begin{array}{l}\text { Brackish water } \\
(\approx 0.5 \% \mathrm{NaCl})\end{array}$ & $\begin{array}{l}\text { Brine }(6 \% \\
\mathrm{NaCl})\end{array}$ & 20.3 & 15.2 & 8.6 & $\begin{array}{l}\text { Pre-treated flat sheet TFC membrane } \\
\text { based on nanofiber substrate }\end{array}$ & 2013 & $\begin{array}{l}\text { Song } \\
\text { et al. } \\
{[22]}\end{array}$ \\
\hline $\begin{array}{c}\text { DI water } \\
(0.0 \% \mathrm{NaCl})\end{array}$ & $\begin{array}{l}\text { Brine }(6 \% \\
\mathrm{NaCl})\end{array}$ & 26.8 & 12.0 & 8.9 & $\begin{array}{l}\text { TFC hollow fiber membrane with } \\
\text { modified support layer }\end{array}$ & 2013 & $\begin{array}{l}\text { Han et } \\
\text { al. } \\
{[24]}\end{array}$ \\
\hline $\begin{array}{l}\text { Waste water } \\
(\approx 0.5 \% \mathrm{NaCl})\end{array}$ & $\begin{array}{l}\text { Brine }(6 \% \\
\mathrm{NaCl})\end{array}$ & 20.8 & 16.0 & 9.2 & $\begin{array}{l}\text { TFC hollow fiber membrane with } \\
\text { modified support layer }\end{array}$ & 2013 & $\begin{array}{l}\text { Han et } \\
\text { al. } \\
{[24]}\end{array}$ \\
\hline $\begin{array}{c}\text { River water } \\
(<0.05 \% \mathrm{NaCl})\end{array}$ & $\begin{array}{l}\text { Brine }(6 \% \\
\mathrm{NaCl})\end{array}$ & 24.3 & 15.2 & 10.3 & $\begin{array}{l}\text { Pre-treated flat sheet TFC membrane } \\
\text { based on nanofiber substrate }\end{array}$ & 2013 & $\begin{array}{l}\text { Song } \\
\text { et al. } \\
{[22]}\end{array}$ \\
\hline $\begin{array}{c}\text { River water } \\
(<0.05 \% \mathrm{NaCl})\end{array}$ & $\begin{array}{l}\text { Brine (6\% } \\
\quad \mathrm{NaCl})\end{array}$ & 25.3 & 15.2 & 10.7 & $\begin{array}{l}\text { Pre-treated flat sheet TFC membrane } \\
\text { based on nanofiber substrate }\end{array}$ & 2013 & $\begin{array}{l}\text { Song } \\
\text { et al. } \\
{[22]}\end{array}$ \\
\hline $\begin{array}{c}\text { River water } \\
(<0.05 \% \mathrm{NaCl})\end{array}$ & $\begin{array}{l}\text { Brine }(6 \% \\
\mathrm{NaCl})\end{array}$ & 24.8 & 16.0 & 11.0 & $\begin{array}{l}\text { TFC hollow fiber membrane with } \\
\text { modified support layer }\end{array}$ & 2013 & $\begin{array}{l}\text { Han et } \\
\text { al. } \\
{[24]}\end{array}$ \\
\hline $\begin{array}{l}\text { Brackish water } \\
(\approx 0.5 \% \mathrm{NaCl})\end{array}$ & $\begin{array}{l}\text { Brine (6\% } \\
\quad \mathrm{NaCl})\end{array}$ & 27.0 & 15.2 & 11.4 & $\begin{array}{l}\text { Pre-treated flat sheet TFC membrane } \\
\text { based on nanofiber substrate }\end{array}$ & 2013 & $\begin{array}{l}\text { Song } \\
\text { et al. } \\
{[22]}\end{array}$ \\
\hline $\begin{array}{c}\text { DI water } \\
(0.0 \% \mathrm{NaCl})\end{array}$ & $\begin{array}{l}\text { Brine (6\% } \\
\quad \mathrm{NaCl})\end{array}$ & 28.9 & 15.0 & 12.0 & $\begin{array}{l}\text { Pre-treated TFC flat sheet membrane } \\
\text { with modified support layer }\end{array}$ & 2013 & $\begin{array}{l}\text { Han et } \\
\text { al. } \\
{[19]}\end{array}$ \\
\hline $\begin{array}{c}\text { DI water } \\
(0.0 \% \mathrm{NaCl})\end{array}$ & $\begin{array}{l}\text { Brine }(6 \% \\
\mathrm{NaCl})\end{array}$ & 19.0 & 25.0 & 13.2 & $\begin{array}{c}\text { TFC membrane with an embedded } \\
\text { woven mesh supported by tricot fabric } \\
\text { feed spacers }\end{array}$ & 2013 & $\begin{array}{l}\text { Straub } \\
\text { et al. } \\
{[20]}\end{array}$ \\
\hline $\begin{array}{c}\text { DI water } \\
(0.0 \% \mathrm{NaCl})\end{array}$ & $\begin{array}{l}\text { Brine }(6 \% \\
\mathrm{NaCl})\end{array}$ & 31.4 & 16.0 & 14.0 & $\begin{array}{l}\text { TFC hollow fiber membrane with } \\
\text { modified support layer }\end{array}$ & 2013 & $\begin{array}{l}\text { Han et } \\
\text { al. } \\
{[24]}\end{array}$ \\
\hline
\end{tabular}




\begin{tabular}{|c|c|c|c|c|c|c|c|}
\hline $\begin{array}{l}\text { River water } \\
(<0.05 \% \mathrm{NaCl})\end{array}$ & $\begin{array}{l}\text { Brine }(6 \% \\
\mathrm{NaCl})\end{array}$ & 36.0 & 15.2 & 15.2 & $\begin{array}{l}\text { Pre-treated flat sheet TFC membrane } \\
\text { based on nanofiber substrate }\end{array}$ & 2013 & $\begin{array}{l}\text { Song } \\
\text { et al. } \\
{[22]}\end{array}$ \\
\hline $\begin{array}{c}\text { DI water } \\
(0.0 \% \mathrm{NaCl})\end{array}$ & $\begin{array}{l}\text { Brine }(6 \% \\
\mathrm{NaCl})\end{array}$ & 50.2 & 15.0 & 20.9 & $\begin{array}{l}\text { TFC polyethermide hollow fiber } \\
\text { membrane with a sponge-like support } \\
\text { structure }\end{array}$ & 2013 & $\begin{array}{l}\text { Chou } \\
\text { et al. } \\
{[25]}\end{array}$ \\
\hline $\begin{array}{c}\text { DI water } \\
(0.0 \% \mathrm{NaCl})\end{array}$ & $\begin{array}{l}\text { Brine } \\
(12 \% \\
\mathrm{NaCl})\end{array}$ & 34.0 & 41.0 & 38.7 & $\begin{array}{l}\text { TFC membrane with an embedded } \\
\text { woven mesh supported by tricot fabric } \\
\text { feed spacers }\end{array}$ & 2013 & $\begin{array}{l}\text { Straub } \\
\text { et al. } \\
{[20]}\end{array}$ \\
\hline $\begin{array}{c}\text { DI water } \\
(0.0 \% \mathrm{NaCl})\end{array}$ & $\begin{array}{l}\text { Brine } \\
(18 \% \\
\mathrm{NaCl})\end{array}$ & 45.0 & 48.0 & 60.0 & $\begin{array}{l}\text { TFC membrane with an embedded } \\
\text { woven mesh supported by tricot fabric } \\
\text { feed spacers }\end{array}$ & 2013 & $\begin{array}{l}\text { Straub } \\
\text { et al. } \\
{[20]}\end{array}$ \\
\hline $\begin{array}{c}\text { DI water } \\
(0.0 \% \mathrm{NaCl})\end{array}$ & $\begin{array}{l}\text { Brine }(6 \% \\
\mathrm{NaCl})\end{array}$ & 36.0 & 24.3 & 24.3 & $\begin{array}{l}\text { TFC polyethersulfone, hollow fiber } \\
\text { membrane with a sponge-like porous } \\
\text { structure }\end{array}$ & 2014 & $\begin{array}{l}\text { Zhang } \\
\text { et al. } \\
\text { [21] }\end{array}$ \\
\hline
\end{tabular}

\section{Implementation of Osmotic Power - Australian Examples}

Sharing the same vision as many European countries, Australia is determined to have a significant part of the nation's electricity production provided by renewable sources in the near future [26] and to gradually reduce its dependence upon fossil fuels. The Renewable Energy Target (RET) [27], the Clean Energy Plan [28] and the proposed introduction of a carbon emissions reduction target by 2020 are believed to be the main drivers of this growth in renewable energy in the country [26]. Through the RET, for example, the Australian Government has established a target of $20 \%$ of Australia's electricity to be sourced from renewable energy by 2020. This represents a significant growth compared to the current share of renewable resources of only $7 \%$. This target is intended to be achieved by a subsidy to renewables that is funded by a tax on non-renewable generations, plus government investments in research and development in the renewable energy sectors [27]. Additionally, the Victorian Renewable Energy Target (VRET) will also be a driver of the increase in renewable energy production in Australia, by requiring $10 \%$ of total electricity generation in Victoria to be sourced from renewable energy sources by 2016.

The current share of renewable energy production in Australia is dominated by hydro energy (63.4\%), followed by wind (22.9\%), bio-energy (11.5\%) and solar energy (2.1\%) [29]. The rest of the share is accounted for by wave, tidal and geothermal energies. It can be postulated that Australia is a country with a large potential for salinity gradient energy due to the existence of various natural solutions that could be combined to extract the salinity gradient energy between them. The possible combinations of solutions and estimates of power productions using the PRO technology are discussed in this section. Although the examples focus on Australia, the possibilities for PRO implementation can be expanded to any other countries presenting similar 
conditions. The possibilities have been selected based primarily on the availability of two natural solutions with different salt concentrations that could be paired for the extraction of the salinity gradient, as presented in Table 4. At the end of this chapter, in Table 6, a clear comparison of each mode is presented in terms of advantages and disadvantages of each PRO mode suggested in this chapter.

Table 4. Combination of solutions for generation of osmotic power - Australian examples

\begin{tabular}{|c|c|c|c|c|c|c|}
\hline $\begin{array}{l}\text { Section } \\
\text { of this } \\
\text { article }\end{array}$ & $\begin{array}{l}\text { Feed solution } \\
\text { (average salt } \\
\text { concentration and } \\
\text { osmotic pressure) }\end{array}$ & $\begin{array}{l}\text { Draw solution } \\
\text { (average salt } \\
\text { concentration and } \\
\text { osmotic pressure) }\end{array}$ & $\begin{array}{l}\text { Water } \\
\text { flux, } J \\
\left(\mathrm{~L} \mathrm{~m} \mathrm{~m}^{-2}\right. \\
\left.\mathrm{h}^{-1}\right)^{1}\end{array}$ & $\begin{array}{l}\text { Achievable } \\
\text { power } \\
\text { density } \\
\left(\mathrm{W} \mathrm{m}^{-2}\right)^{2}\end{array}$ & $\begin{array}{c}\text { Power } \\
\text { production } \\
\text { potential per } \mathrm{m}^{3} \\
\mathrm{~s}^{-1} \text { of feed } \\
\text { solution } \\
(\mathrm{MW})^{3} \\
\end{array}$ & $\begin{array}{l}\text { Australian example (feed } \\
\text { solution vs. draw solution) and } \\
\text { osmotic power estimate }\end{array}$ \\
\hline \multirow{2}{*}{5.1} & \multirow{2}{*}{$\begin{array}{l}\text { River water }(< \\
0.05 \%, \approx 0 \text { bar })\end{array}$} & \multirow{2}{*}{$\begin{array}{c}\text { Sea water }(\approx 3.5 \% \text {, } \\
28.5 \text { bar })\end{array}$} & \multirow{2}{*}{21.3} & \multirow{2}{*}{8.4} & \multirow{2}{*}{1.4} & $\begin{array}{l}\text { Brisbane River vs. sea water, } \\
10 \mathrm{MW}^{4}\end{array}$ \\
\hline & & & & & & $\begin{array}{c}\text { Hinze Dam vs. Sea water, } 2.9 \\
\text { MW }^{5}\end{array}$ \\
\hline \multirow[b]{2}{*}{5.2} & \multirow[b]{2}{*}{$\begin{array}{c}\text { Sea water }(\approx 3.5 \% \text {, } \\
28.5 \text { bar })\end{array}$} & \multirow{2}{*}{$\begin{array}{l}\text { Concentrated brine } \\
\text { from salt lakes }(\approx \\
32 \%, 260 \text { bar })\end{array}$} & \multirow[b]{2}{*}{174} & \multirow[b]{2}{*}{560} & \multirow[b]{2}{*}{11.6} & $\begin{array}{l}\text { Sea water } v \text { s. brine from Lake } \\
\text { Torrens, } 2.6 \mathrm{GW}^{6}\end{array}$ \\
\hline & & & & & & $\begin{array}{l}\text { Sea water } v \text { s. brine from Lake } \\
\text { Torrens and Lake Eyre } \\
\text { combined, } 11.6 \mathrm{GW}^{7}\end{array}$ \\
\hline 5.3 & $\begin{array}{l}\text { Brackish } \\
\text { groundwater from } \\
\text { the mining industry } \\
(\approx 1.0 \%, 8 \text { bar })\end{array}$ & $\begin{array}{l}\text { Concentrated brine } \\
\text { from the brackish } \\
\text { groundwater }(\approx 8 \% \text {, } \\
65 \text { bar })^{8}\end{array}$ & 43.3 & 34.8 & 2.9 & $\begin{array}{l}\text { Sea water vs. concentrated } \\
\text { brine from coal seam gas water } \\
\text { from the Surat and Bowen } \\
\text { Basins, } 22 \mathrm{MW}^{9}\end{array}$ \\
\hline 5.3 & $\begin{array}{l}\text { River water }(< \\
0.05 \%, \approx 0 \text { bar })\end{array}$ & $\begin{array}{l}\text { Concentrated brine } \\
\text { from the brackish } \\
\text { groundwater }(\approx 8 \% \text {, } \\
65 \text { bar })^{8}\end{array}$ & 81.3 & 73.5 & 3.3 & $\begin{array}{l}\text { River water vs. concentrated } \\
\text { brine from coal seam gas water } \\
\text { from the Surat and Bowen } \\
\text { Basins, } 33 \mathrm{MW}^{10}\end{array}$ \\
\hline 5.3 & $\begin{array}{l}\text { Brackish } \\
\text { groundwater }(\approx \\
1.0 \%, 8 \text { bar })\end{array}$ & $\begin{array}{l}\text { Hypersaline } \\
\text { groundwater }(\approx \\
20 \%, 160 \text { bar })\end{array}$ & 116 & 250 & 7.7 & $\begin{array}{l}\text { Brackish groundwater vs. } \\
\text { hypersaline groundwater from } \\
\text { gold mining sites in Western } \\
\text { Australia, } 77 \mathrm{MW}^{11}\end{array}$ \\
\hline 5.3 & $\begin{array}{l}\text { River water }(< \\
0.05 \%, \approx 0 \text { bar })\end{array}$ & $\begin{array}{l}\text { Hypersaline } \\
\text { groundwater }(\approx \\
20 \%, 160 \text { bar })\end{array}$ & 122 & 276 & 8.1 & $\begin{array}{l}\text { River water vs. hypersaline } \\
\text { groundwater from gold mining } \\
\text { sites in Western Australia, } 81 \\
\text { MW }^{10}\end{array}$ \\
\hline \multirow[b]{2}{*}{5.4} & \multirow[b]{2}{*}{$\begin{array}{l}\text { Sea water }(\approx 3.5 \% \text {, } \\
28.5 \text { bar })\end{array}$} & \multirow[b]{2}{*}{$\begin{array}{l}\text { Concentrated brine } \\
\text { from desalination } \\
\text { plants }(\approx 6 \%, 48.8 \\
\text { bar })\end{array}$} & \multirow[b]{2}{*}{15.3} & \multirow[b]{2}{*}{4.3} & 1.1 & $\begin{array}{l}\text { Sea water vs. brine from the } \\
\text { Gold Coast Desalination Plant, } \\
1 \mathrm{MW}^{13}\end{array}$ \\
\hline & & & & & $1.1 / 1.4$ & $\begin{array}{l}\text { Sea water vs. brine from the } \\
\text { Gold Coast Desalination Plant } \\
\text { (for } 3 \text { months) and Reservoir } \\
\text { water vs. brine from the Gold } \\
\text { Coast Desalination Plant (for } 9 \\
\text { months), } 2.3 \mathrm{MW}^{13}\end{array}$ \\
\hline 5.5 & $\begin{array}{l}\text { Waste water }(\approx \\
0.1 \%, 0.8 \text { bar })\end{array}$ & $\begin{array}{l}\text { Sea water }(\approx 3.5 \% \text {, } \\
28.5 \text { bar })\end{array}$ & 20.8 & 8.0 & 1.4 & $\begin{array}{c}\text { Luggage Point waste water } \\
\text { (Brisbane) vs. sea water, } 3 \\
\text { MW }^{14}\end{array}$ \\
\hline 5.6 & $\begin{array}{l}\text { Waste water }(\approx \\
0.1 \%, 0.8 \text { bar })\end{array}$ & $\begin{array}{l}\text { Concentrated brine } \\
\text { from desalination } \\
\text { plants }(\approx 6 \%, 48.8 \\
\text { bar })\end{array}$ & 36.0 & 24.0 & 2.4 & $\begin{array}{c}\text { Luggage Point waste water } \\
\text { (Brisbane) vs. brine from the } \\
\text { projected desalination plant, } 5 \\
\text { MW }^{14}\end{array}$ \\
\hline \multicolumn{7}{|c|}{$\begin{array}{l}{ }^{1} \text { Water flux, } J \text { : calculated using Eq. } 1 \text {, assuming intrinsic water permeability }=1.5 \mathrm{~L} \mathrm{~m}^{-2} \mathrm{~h}^{-1} \mathrm{bar}^{-1} \text { [4], and neglecting the effectsofECP,ICF } \\
\text { and reverse flux of salts. } \\
{ }^{2} \text { Power density: given by the product of the operating pressure, } P \text {, and the water flux, } J \text {. The operat ing pressure was assumed as half the } \\
\text { osmotic pressure differential, as suggest ed in the literature }[2,4,12,14] \text {. } \\
{ }^{3} \text { Power per } \mathrm{m}^{3} \mathrm{~s}^{-1} \text { of feed solution: given by theproduct of the operating pressure and the unit flow of feed solution. } \\
{ }^{4} \text { Assuming the Q Q } \mathrm{Q}_{70} \text { of the Brisbane River }\left(7 \mathrm{~m}^{3} \mathrm{~s}^{-1} \text { ) }\right. \\
{ }^{5} \text { Assuming the Hinze Dam provides a fresh water flow }=2 \mathrm{~m}^{3} \mathrm{~s}^{-1} \text {. } \\
\text { Assumed seawater flow }=225 \mathrm{~m}^{3} \mathrm{~s}^{-1}[30] \text {. }\end{array}$} \\
\hline
\end{tabular}


${ }_{9}^{8}$ Assuming that the groundwat er is transferred to evaporation ponds, where it reaches a salinity level of around $8 \%$.

${ }^{9}$ Using half of the estimated product waterflow in the Surat and Bowen Basins (i.e., $7.6 \mathrm{~m}^{3} \mathrm{~s}^{-1}$ ).

${ }^{10}$ Assumed river flow $=10 \mathrm{~m}^{3} \mathrm{~s}^{-1}$.

${ }_{11}$ Assumed groundwater flow $=10 \mathrm{~m}^{3} \mathrm{~s}^{-1}$.

12 Assumed seawater flow $=0.9 \mathrm{~m}^{3} \mathrm{~s}^{-1}$ (same magnitude as the brineflow)

${ }^{13}$ Assumed seawater flow $=0.9 \mathrm{~m}^{3} \mathrm{~s}^{-1}$ (same magnitude as the brineflow). Assuming the Hinze Dam provides a fresh water flow $=2 \mathrm{~m}^{3} \mathrm{~s}^{-1}$.

${ }^{14}$ Assumed discharge of waste water $=2.1 \mathrm{~m}^{3} \mathrm{~s}^{-1}[31]$.

\subsection{River water vs. sea water}

Feed solution: river water

Draw solution: sea water

The first combination of solutions that will be discussed in this article is river water and sea water. This is perhaps the most realistic combination of solutions for the implementation of large-scale PRO plants in the short and mid-terms. This is due to the rapid advancements in PRO membrane development, and to the existence of the already operating river water $v s$. sea water prototype plant (Norway), which has been acquiring all the necessary know-how related to osmotic power production using these types of solutions.

A general sketch of an osmotic power plant operating on the basis of these two solutions is illustrated in Figure 3. Assuming a salt concentration close to zero for the feed solution (river water) and a salt concentration around 3.5\% for the draw solution (sea water), this combination would provide and osmotic pressure differential of approximately 28 bar. Using a value of 1.5 $\mathrm{L} \mathrm{m}^{-2} \mathrm{~h}^{-1}$ bar $^{-1}$ for membrane permeability, which represents an average permeability of conventional thin-film composite RO membranes [4], and neglecting the detrimental effects of concentration polarization, the resulting water flux is $21.3 \mathrm{~L} \mathrm{~m}^{-2} \mathrm{~h}^{-1}$. Considering an operating pressure of about one half of the osmotic pressure difference [2, 4, 12, 14], the potential power output from this scheme is $1.4 \mathrm{MW}$ per $\mathrm{m}^{3} \mathrm{~s}^{-1}$ of feed solution (i.e., river water), and the resulting membrane power density is $8.4 \mathrm{~W} \mathrm{~m}^{-2}$. This is a very conservative estimate, since it is based on characteristics of commercially available membranes. It should be noted that higher values for membrane permeability and water flux have already been observed under laboratory conditions for membranes designed specifically for PRO application (refer to Table 2).

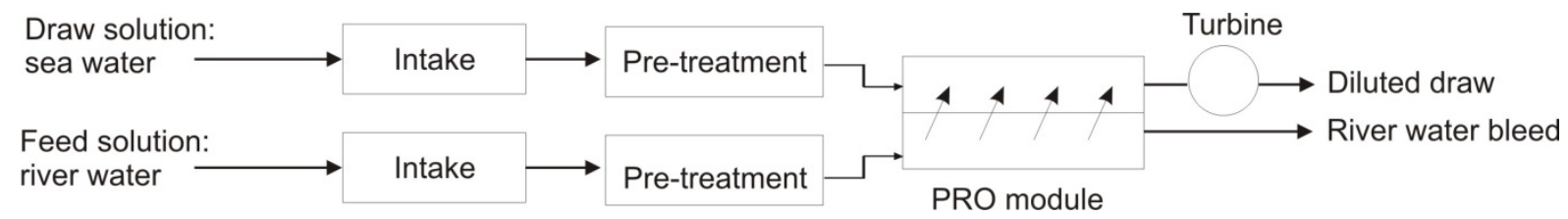

Figure 3. A conceptual scheme for a PRO plant using river water (feed) and sea water (draw) 
Like Norway, Australia would also have similar sites where the installation of osmotic power plants based on river and sea waters would be feasible. Some relevant differences in relation to most European rivers would be the seasonality of the power production in Australia, given the high temporal variability of its rivers. Moreover, as Australian rivers are significantly affected by tidal conditions, the point of extraction of the river water would have to be located further upstream, where the tide has no or little influence on the river salinity. This would possibly incur additional costs related to the channelling and transport of the river water into the plant.

The Brisbane River, located in Queensland, can be used as an example for the calculation of osmotic power. This river has a $\mathrm{Q}_{70}$ flow (flow that is equalled or exceeded during $70 \%$ of the time) of $7 \mathrm{~m}^{3} \mathrm{~s}^{-1}$ [32]. If we consider a power production of $1.4 \mathrm{MW}$ per $\mathrm{m}^{3} \mathrm{~s}^{-1}$ of river water, this would translate into a power production of 9.8 MW, making it competitive with other renewable energy plants located in Queensland, such as wind, solar and biomass [33]. The financial viability of this plant would depend largely on the power density of the membranes, as the greatest part of the capital cost would be related to the cost of membrane installation. Commercially available membranes designed for FO and RO have a power output ranging from 0.3 to $2.7 \mathrm{~W} \mathrm{~m}^{-2}$ (refer to Table 2). Modified membranes for PRO application have been able to produce up to $11.5 \mathrm{~W} \mathrm{~m}^{-2}$ [21] under laboratory conditions. It is highly argued in the literature that the minimum power density to make osmotic power financially viable is $5 \mathrm{~W} \mathrm{~m}^{-}$ 2. With this power output, a total membrane area of $1.96 \mathrm{~km}^{2}$ would be required for an osmotic power plant located at the Brisbane River.

The cost of membrane installation of a large Australian desalination plant, located in Perth $\left(\right.$ capacity $=144 \mathrm{ML}$ day ${ }^{-1}$ ), was approximately AU\$ $452 \mathrm{~m}^{-2}$. This was estimated based on the reported desalination plant capital cost (AU\$ 317 million) and the installed membrane area $(\approx$ $700,000 \mathrm{~m}^{2}$ ) [34]. Applying this unit cost to an osmotic power plant, the total capital cost for $1.96 \mathrm{~km}^{2}$ of membranes would be around AU\$ 885 million, or AU\$ 90,000 kW-1. Just for comparison, traditional renewable energy plants, such as wind and solar have much lower costs. Solar farms have a capital cost of between AU\$ 6,800 and AU\$ 7,700 $\mathrm{kW}^{-1}$ [35]. Wind energy has a capital cost varying from AU\$ 2,000 to AU\$ 2,500 $\mathrm{kW}^{-1}$ [36]. More recent figures from the desalination industry on capital costs per unit area of membrane, however, show that the cost of membrane installation is between AU\$ 20 and AU\$ $40 \mathrm{~m}^{-2}$ [37]. If these figures were used to estimate the capital cost of an osmotic power plant, the unit cost would fall 
between AU\$ 4,000 and AU\$ 8,000 $\mathrm{kW}^{-1}$. This demonstrates that osmotic power has the potential to become competitive with other traditional types of renewable energy, and even more economical than other sources of ocean energy, such as tidal and wave [38].

\subsubsection{Energy production during idle periods of desalination plants (using same structure)}

Current desalination technologies, such as reverse osmosis are expensive and energy intensive. On average, the production of $1 \mathrm{~m}^{3} \mathrm{~s}^{-1}$ of fresh water from sea water via RO requires 12,000 $\mathrm{kW}$ of power [39]. On the other hand, $1 \mathrm{~m}^{3} \mathrm{~s}^{-1}$ of fresh water can generate around 1,400 kW of power under PRO conditions (refer to Table 4). In Australia, the growing demands for potable water have been driving momentum to construction of new desalination plants. In 2009, for instance, the freshwater production capacity of Australian desalination plants was 294,000 $\mathrm{m}^{3}$ day $^{-1}$, distributed over more than 45 plants utilizing RO [39]. The capacity of the RO desalination plants under construction in 2009 was 976,000 $\mathrm{m}^{3}$ day ${ }^{-1}$ and the estimated capacity for the projected plants is $925,000 \mathrm{~m}^{3}$ day $^{-1}$ [39]. These facilities are usually built as insurance, to guarantee the water supply during times of water scarcity. This means that these plants remain idle for large periods of time, incurring high maintenance costs.

It has been suggested in the literature that osmotic power could be possibly produced within the same desalination facility, using the same membrane infrastructure, during periods when the plant is not producing potable water $[4,40]$, as illustrated in Figure 4.

a) Desalination plant in operation

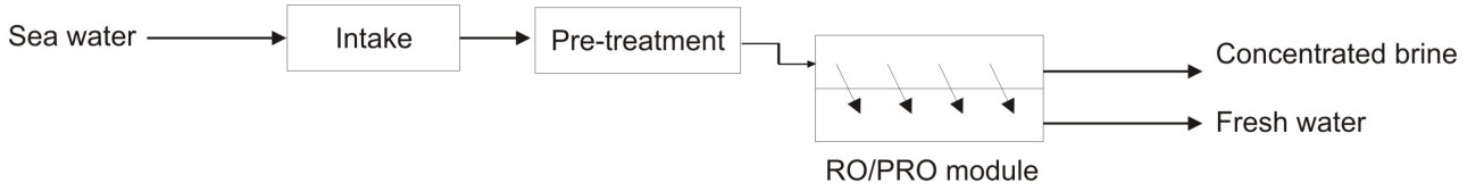

b) Desalination plant idle

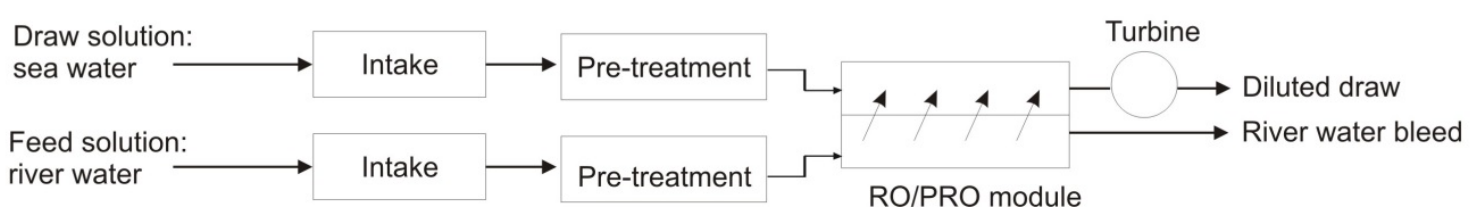

Figure 4. A conceptual scheme for a SWRO desalination plant that is converted to an osmotic power plant during idle periods of operation 
The Gold Coast Desalination Plant, located in Queensland, Australia, was designed and built in 2007 to produce $125,000 \mathrm{~m}^{3}$ day $^{-1}$ of potable water [41] to provide water insurance to the growing population in the Brisbane region. As the availability of water from conventional sources has been reliable over the past years, the plant has been put on stand-by mode since 2010. It has been operating only for sporadic periods, such as during the flood events in January 2011 in Brisbane. Even during that time, however, the production of water by the plant was much less than $50,000 \mathrm{~m}^{3}$ day $^{-1}$ [42], meaning that the plant was operating at less than $40 \%$ of the total installed capacity. Using system dynamics modelling, it was demonstrated that the infrastructure utilization of the Gold Coast Desalination Plant could be increased from the current efficiency of $25 \%$ to $91 \%$ [4, 40] if osmotic power production was added to the system.

Assuming that the plant operates for three months of the year at 50,000 $\mathrm{m}^{3}$ day $^{-1}$, the total energy required for the desalination process will be approximately 15 GWh per year. From data presented in the literature about the membrane elements used at the Gold Coast Desalination Plant [43-45], it can be worked out that an area of approximately 630,000 $\mathrm{m}^{2}$ of membranes is installed in the plant. If these membrane modules were replaced by dual-purpose membranes, and considering that the power production per unit area of membrane will eventually reach $8.4 \mathrm{~W} \mathrm{~m}^{-2}$ (refer to Table 4), the power production capacity would be 5,292,000 W (5.3 MW), meaning the generation of 34 GWh of energy. This is much higher than the energy consumed in the desalination process. To keep the power production at maximum, however, a flow of fresh water of around $3.8 \mathrm{~m}^{3} \mathrm{~s}^{-1}$ would have to be fed into the plant. Freshwater sources include nearby streams and a local water supply reservoir (Hinze Dam) located $30 \mathrm{~km}$ from the Gold Coast Desalination Plant. Using monthly average data for inflows, withdrawals, evaporation and precipitation of Hinze Dam [46], it can be estimated that this reservoir could provide $2 \mathrm{~m}^{3} \mathrm{~s}^{-1}$ of fresh water to the plant without compromising the water supply for the population. Assuming a power outcome of $1.4 \mathrm{MW}$ per $\mathrm{m}^{3} \mathrm{~s}^{-1}$ (refer to Table 4), this volume could generate 2.8 MW of power. Over nine months, this would translate into a total energy of $18 \mathrm{GWh}$, which is substantial enough to offset the energy consumption by the desalination plant.

The success of such a scheme would be predicated on the development of membrane modules that are suitable for both desalination (reverse osmosis) and osmotic power (pressure retarded osmosis), something that appears to be technically viable, although not yet available. Another precondition would be the existence of a source of fresh water, such as a river or a reservoir, 
to feed the power plant. It would of course be counterintuitive to utilise a desalination plant to generate electricity in times of water shortage, as this would further contribute to water scarcity. As discussed, the main hurdle to overcome before the implementation of this symbiotic relationship is the development of a membrane that could be efficiently used for both desalination and power generation. Advocates of osmotic power have already suggested that research and development of osmotic power membranes should begin to focus on the possibility of employing the same membrane in both power and desalination processes $[47,48]$

For existing desalination plants, the transition from the already installed membrane modules to the new, double-purpose modules would incur additional costs. For new desalination plants, however, the possibility of making it a dual-purpose facility should be taken into consideration in the project phase.

\subsection{Sea water vs. concentrated brine from salt lakes}

Feed solution: sea water

Draw solution: concentrated brine from salt lakes

This proposed scheme is based on sea water as feed solution and concentrated brine from salt lakes as draw solution (refer to Figure 5). Australian salt lakes have already been suggested in the literature [30, 49] for generation of osmotic power. This idea involves pumping sea water into the salt lake and letting the volume of water in the lake decrease due to evaporation, consequently increasing the salt concentration in the water. This hyper-concentrated solution would then be paired with sea water (less concentrated solution) in a PRO plant. Technically, this scheme is still conceptual due to the inexistence in the market of appropriate membranes that would be able to withstand the high osmotic pressure differentials between these solutions. However, a few studies have already investigated the use of solutions with high salt concentrations. For example, She et al. [16] reported a power density of $6.7 \mathrm{~W} \mathrm{~m}^{-2}$ for a commercial FO cellulose triacetate membrane supported by a woven fabric. Straub et al. [20] investigated a customised thin-film composite membrane with an embedded woven mesh, supported by tricot fabric feed spacers, that was able to withstand high applied pressures. The power outcome was $38.7 \mathrm{~W} \mathrm{~m}^{-2}$ when using pure water as the feed solution and $12 \% \mathrm{NaCl}$ brine as the draw solution. A power density of $60 \mathrm{~W} \mathrm{~m}^{-2}$ was achieved for an $18 \% \mathrm{NaCl}$ draw solution. 


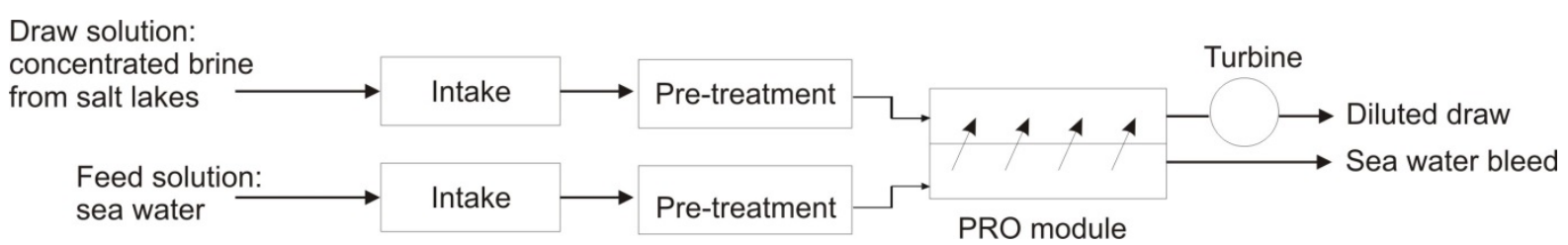

Figure 5. A conceptual scheme for a PRO plant based on sea water (feed) and concentrated brine from salt lakes (draw)

Loeb [8], in 1975, was the first to suggest that salt lakes, such as the Dead Sea and the Great Salt Lake, could be potential sources of brine for osmotic power generation. Wick [3], in 1978, suggested that using salt domes as a brine source in combination with fresh water would be even more cost-effective because the concentration of the salt pans could be maintained even higher than that of the Dead Sea or Great Salt Lake by controlled influx of sea water. Recently, Kelada [49] published a list of hypersaline sites and their conjugated low salinity waters that could be used as sources of brine for the production of osmotic power around the world. These are summarised in Table 5 .

As seen in Table 5, Lake Torrens and Lake Eyre, located in South Australia, have been suggested as possible locations for the implementation of an osmotic power plant [30, 49]. Lake Torrens has a surface area of $5,700 \mathrm{~km}^{2}$ and remains naturally dry for almost the entire year. Mean rainfall ranges from $200 \mathrm{~mm}$ to $250 \mathrm{~mm}$ and annual potential evaporation is around $3,500 \mathrm{~mm}$ in the region of the lake. It is suggested that a seawater canal of $1,000 \mathrm{~m}^{3} \mathrm{~s}^{-1}$ capacity could be extended from the Spencer Gulf, located about $80 \mathrm{~km}$ south of the southern end of the lake to fill in the lake [49]. The idea of permanently filling in Lake Torrens and Lake Eyre (another dry lake located north of Lake Torrens) from the Spencer Gulf had already been considered by the Australian Government with the purpose of improving the local climate, but the project was rejected. The idea is being considered again due to the predictions of climate models that the interior of southern Australia will become drier than at present, affecting agricultural activities more severely. It is believed that filling in these two lakes will increase rainfall in the region because of the increased evaporation from the lakes' waters. 
Table 5. Natural sources of highly concentrated solutions and conjugated sources of feed solutions for the production of osmotic power

\begin{tabular}{|c|c|c|c|c|c|c|}
\hline $\begin{array}{l}\text { Suggested draw } \\
\text { solution source }\end{array}$ & $\begin{array}{c}\text { Salt } \\
\text { concentration in } \\
\text { the draw solution } \\
(\%)\end{array}$ & $\begin{array}{l}\text { Conjugated feed } \\
\text { solution source }\end{array}$ & $\begin{array}{c}\text { Salt } \\
\text { concentration in } \\
\text { the feed solution } \\
\text { (\%) }\end{array}$ & $\begin{array}{l}\text { Suggesting } \\
\text { Source }\end{array}$ & $\begin{array}{l}\text { Approximate } \\
\text { osmotic pressure } \\
\text { difference, } \Delta \pi \\
\text { (bar) }\end{array}$ & $\begin{array}{c}\text { Power } \\
\text { Output } \\
\left(\mathrm{MW} \text { per } \mathrm{m}^{3}\right. \\
\mathrm{s}^{-1} \text { of feed } \\
\text { solution })^{1}\end{array}$ \\
\hline $\begin{array}{c}\text { Great Salt Lake, } \\
\text { USA }\end{array}$ & 24 & $\begin{array}{l}\text { Bear, Weber or } \\
\text { Jordan Rivers }\end{array}$ & $<0.1$ & $\begin{array}{c}\text { Loeb }[8,50], \\
\text { Wick [3], } \\
\text { Kelada [49, } \\
51],\end{array}$ & 200 & 10.0 \\
\hline $\begin{array}{l}\text { Lake Torrens, } \\
\text { Australia, Phase } \\
\text { I }\end{array}$ & 32 & Indian Ocean & 3.5 & Kelada [30, 49] & 240 & 12.0 \\
\hline $\begin{array}{l}\text { Lake Torrens, } \\
\text { Australia, Phase } \\
\text { II }\end{array}$ & 32 & Indian Ocean & 3.5 & Kelada [30, 49] & 240 & 12.0 \\
\hline $\begin{array}{c}\text { Lake Eyre, } \\
\text { Australia }\end{array}$ & 33 & Indian Ocean & 3.5 & Kelada $[30,49]$ & 250 & 12.4 \\
\hline $\begin{array}{l}\text { Lake Gairdner, } \\
\text { Australia }\end{array}$ & $\begin{array}{l}\text { Salt bed } \\
\text { (variable) }\end{array}$ & Indian Ocean & 3.5 & Kelada [49] & N/A & N/A \\
\hline $\begin{array}{c}\text { Sebjet Tah, } \\
\text { Western Sahara }\end{array}$ & $\begin{array}{l}\text { Lowland } \\
\text { (variable) }\end{array}$ & Atlantic Ocean & 3.5 & Kelada [49] & N/A & N/A \\
\hline $\begin{array}{l}\text { Lake Assal, } \\
\text { Djibouti }\end{array}$ & 35 & $\begin{array}{l}\text { Ghoubbet al- } \\
\text { Kharab Hot } \\
\text { Springs }\end{array}$ & $3.5-4.5$ & Kelada [49] & 260 & 13.0 \\
\hline $\begin{array}{l}\text { The Aral Sea, } \\
\text { Kazakhstan }\end{array}$ & 30 & The Caspian Sea & $0.1-0.2$ & Kelada [49] & 250 & 12.5 \\
\hline $\begin{array}{l}\text { Zaliv Kara- } \\
\text { Bogaz-Gol, } \\
\text { Turkmenistan }\end{array}$ & 33 & The Caspian Sea & $1-1.5$ & Kelada [49] & 270 & 13.3 \\
\hline $\begin{array}{l}\text { Lake } \\
\text { Baskunchak, } \\
\text { Russia }\end{array}$ & 30 & $\begin{array}{l}\text { The Volga River } \\
\text { / Caspian Sea }\end{array}$ & $0.1-0.2$ & Kelada [49] & 250 & 12.5 \\
\hline $\begin{array}{c}\text { Chott el Jerid, } \\
\text { Tunisia }\end{array}$ & 32 & $\begin{array}{c}\text { The } \\
\text { Mediterranean }\end{array}$ & 3.5 & Kelada [52] & 240 & 12.0 \\
\hline $\begin{array}{l}\text { Chott Melrhir, } \\
\text { Algeria }\end{array}$ & $\begin{array}{c}\text { Salt bed } \\
\text { (variable) }\end{array}$ & $\begin{array}{c}\text { The } \\
\text { Mediterranean }\end{array}$ & 3.5 & Kelada [49] & N/A & N/A \\
\hline $\begin{array}{l}\text { Qattara } \\
\text { Depression, } \\
\text { Egypt }\end{array}$ & $\begin{array}{r}\text { Lowland } \\
\text { (variable) }\end{array}$ & $\begin{array}{c}\text { The } \\
\text { Mediterranean }\end{array}$ & 3.5 & Kelada [49] & N/A & N/A \\
\hline Lake Urmia, Iran & 33 & $\begin{array}{l}\text { Zarrineh \& } \\
\text { Simineh / } \\
\text { Caspian Sea }\end{array}$ & $<0.1$ & Kelada [49] & 280 & 13.8 \\
\hline $\begin{array}{l}\text { Lake Tuz, } \\
\text { Turkey }\end{array}$ & 33 & $\begin{array}{l}\text { Kizil Irmak } \\
\text { River }\end{array}$ & $<0.1$ & Kelada [49] & 280 & 13.8 \\
\hline $\begin{array}{l}\text { Arabian } \\
\text { Peninsula }\end{array}$ & $\begin{array}{l}\text { Lowland } \\
\text { (variable) }\end{array}$ & $\begin{array}{l}\text { Red Sea, Persian } \\
\text { Gulf }\end{array}$ & 0.5 & Kelada [49] & N/A & N/A \\
\hline $\begin{array}{l}\text { The Dead Sea, } \\
\text { Israel/Jordan }\end{array}$ & 33 & $\begin{array}{l}\text { The } \\
\text { Mediterranena / } \\
\text { The Red Sea }\end{array}$ & $3.5-4.5$ & $\begin{array}{l}\text { Loeb [8, 53], } \\
\text { Wick [3], } \\
\text { Kelada [49] }\end{array}$ & 240 & 12.2 \\
\hline $\begin{array}{l}\text { Gran Bajo de } \\
\text { San Julian }\end{array}$ & 33 & $\begin{array}{l}\text { Atlantic Ocean- } \\
\text { Argentina shore }\end{array}$ & 3.5 & Kelada [49] & 250 & 12.4 \\
\hline $\begin{array}{c}\text { Laguna Salgada, } \\
\text { Mexico }\end{array}$ & $\begin{array}{c}\text { Salt bed } \\
\text { (variable) }\end{array}$ & $\begin{array}{l}\text { Gulf of } \\
\text { California }\end{array}$ & $3.5-4.5$ & Kelada [49] & N/A & N/A \\
\hline
\end{tabular}

${ }^{\mathrm{T}}$ The power output is given by the product of the operating pressure and the unit flow. The operating pressure wasassumedashalftheosmotic pressure differential, as suggested in the literature[2, 4,12,14]

The sea water from the Spencer Gulf would be elevated 35 metres through a pumping system to the southern end of Lake Torrens, where a power generation plant would be installed. The discharged water from the power plant would be transported $160 \mathrm{~km}$ north, and discharged into Lake Torrens, at its northernmost end. The long travelling distance from the north to the south of the lake, together with the high evaporative losses, would allow for the formation of highly 
concentrated brine in the lake. The lake would therefore work as a recycle loop for the power plant. Calculations considering the amount of water disposed of from the power plant and transported into the lake, and evaporation rates, show that the lake could provide brine at 32\% salt concentration, which would be used as the draw solution in the osmotic power plant. The feed solution would be sea water, at approximately 3.5\% salt concentration. The configuration proposed for Lake Torrens could generate up to $2.6 \mathrm{GW}$ for a $225 \mathrm{~m}^{3} \mathrm{~s}^{-1}$ seawater flow rate (refer to Table 4).

Lake Eyre and Lake Torrens were also suggested as a combined source of brine for osmotic power generation [30]. Lake Eyre has a surface area of $9,500 \mathrm{~km}^{2}$, and the evaporation rate from the lake is around $650 \mathrm{~m}^{3} \mathrm{~s}^{-1}$. This high evaporative demand could act as a vacuum pump to dry the lake and to concentrate the sea water that would be diverted from the Spencer Gulf (350 km south of the lake) into the lake. The achievable brine salinity is estimated to be $35 \%$ [30]. The sea water would be diverted into the lake from the Spencer Gulf through a canal that would first run through Lake Torrens. The power plant would be situated at the southern end of Lake Torrens. Unlike the first project, the excess flow from the power plant would be discharged into Lake Eyre South, and the flow would go to Lake Eyre North and then into Lake Torrens. Instead of $75 \mathrm{~m}^{3} \mathrm{~s}^{-1}$ of brine provided through the first project, in this project, $375 \mathrm{~m}^{3}$ $\mathrm{s}^{-1}$ of brine would be provided. The configuration proposed is able to produce over $10 \mathrm{GW}$, with about $1,000 \mathrm{~m}^{3} \mathrm{~s}^{-1}$ flow of sea water diverted into the plant.

As already discussed, these two projects are still conceptual, and the performance of a cost analysis would be required in order to prove their financial viability. They involve the construction of extensive artificial canals, which would make their implementation excessively costly. Neither the sizes of the power plants, nor the availability of membranes for this purpose, are discussed in the above publications. Presumably, osmotic power plants of those magnitudes would utilise an extensive area due to the large amount of membranes that the project would require. Nevertheless, provided that membranes for high osmotic pressure gradients become available at commercial scale and low cost, there is no doubt these projects would be justifiable. Apart from increasing Australia's energy supply by over 20\%, other indirect benefits would be brought about by these projects. For example, drought relief and development is suggested, which could be attractive for the installation of resorts, recreation and fishing villages. Also, the canals proposed for sea water and river diversion would all be navigable, which could help to attract more development to the region. Moreover, the climate of the region would become 
less arid due to the increased precipitation, which could benefit the local agricultural activities. One downside, however, is the distance of the power plant to the energy consumption centres, which would significantly add to the costs of the power generation.

\subsection{Brackish groundwater from the mining industry vs. concentrated brine from brackish groundwater}

Feed solution: brackish groundwater from the mining industry Draw solution: concentrated brine from brackish groundwater

Another source that could be used as draw solution in an osmotic power plant is the concentrated brine formed from the saline groundwater extracted by the mining industry. In this scheme, brackish or saline groundwater would be transferred to evaporation ponds, where the concentration of salt in the solution would be increased as a result of the loss of water to evaporation. This concentrated solution could then be paired with a lower concentrated solution. This scheme is represented in Figure 6.

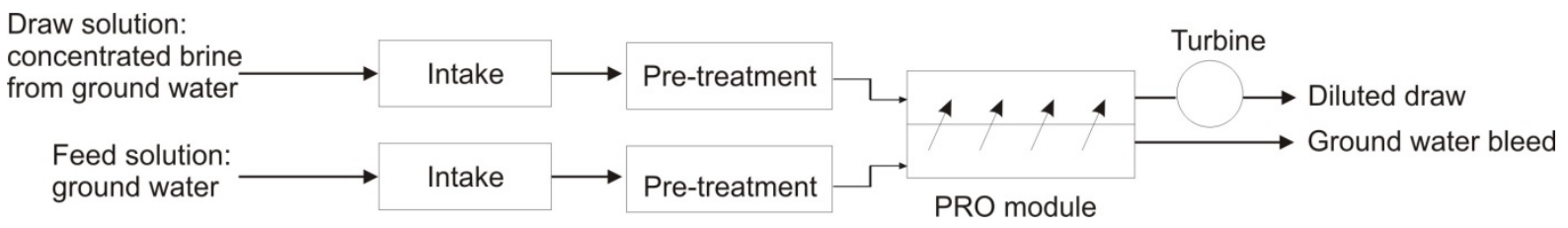

Figure 6. A conceptual scheme for a PRO plant based on brackish groundwater (feed) and concentrated brine from groundwater (draw)

Australia's mining industry utilises great quantities of groundwater as it is more readily available than surface waters and less affected by losses to evaporation. It is estimated that the mining sectors of Western Australia, Queensland and New South Wales are responsible together for the abstraction of $1,130 \mathrm{hm}^{3} \mathrm{yr}^{-1}\left(36 \mathrm{~m}^{3} \mathrm{~s}^{-1}\right)$ of groundwater [54-56], representing $22 \%$ of the total groundwater used in Australia.

Most of the groundwater in Australia contains large quantities of dissolved salts. The saline water is used in mine sites for general mining operations. In the extraction of coal seam gas (CSG), for example, there is a need to 'dewater' the coal seams to depressurise the gas. The large volumes of CSG by-product water gives rise to potential surface issues in the handling, disposal and use of this saline water $[57,58]$. 
CSG production in Australia has been growing fast in recent years. The production is concentrated in Queensland (particularly in the Bowen and Surat basins) and in the northeast of New South Wales. Given the significant reserves in these states, the expansion of the CSG industry is predicted to continue, as demands in the domestic and export markets increase. The annual number of wells drilled in Queensland increased from 10 in the early 1990s to almost 600 in 2010-11 [59]. The CSG industry in the Bowen and Surat basins are responsible for the production of around $480 \mathrm{hm}^{3}$ of water per year $\left(15 \mathrm{~m}^{3} \mathrm{~s}^{-1}\right)$ [58]. The salinity of the by-product water varies from $0.1 \%$ to over $1 \%[57,58]$. The disposal of this water is one of the biggest challenges for the CSG industry. Alternatives include the disposal of water in evaporation ponds, the re-use of water for CSG operations, injection into a non-coal aquifer or the use of water for recreation. After the treatment to reduce the salt concentration, the produced water can also be directly discharged into surface waters to increase the environmental flow, or used for wildlife and livestock watering and agriculture [60].

One alternative for the CSG by-product water could be its utilisation in the production of osmotic power. The water would be transferred to evaporation ponds, where concentrated brine would be formed after losses of water to evaporation. This brine would then be coupled with either channelled sea water or with the less concentrated product water in order to produce osmotic power. The power could be used in the CSG mines, reducing their operation costs and, additionally, their dependability on energy from fossil fuel combustion. Simulations carried out for the Bowen and Surat basins, assuming half of the annual product water (i.e., $240 \mathrm{hm}^{3}$ $\mathrm{yr}^{-1}$ ), 1\% $\mathrm{NaCl}$ concentration, $2260 \mathrm{~mm} \mathrm{yr}^{-1}$ of evaporation rate [61], 215 evaporation ponds with a surface area of 50 hectares each and an average depth of 2 metres, showed that it is possible to generate brine at $8 \%$ salt concentration from the CSG waters. When paired with the by-product water, with approximately $1 \% \mathrm{NaCl}$ concentration, it is possible to generate 2.9 MW of power per unit flow of feed solution (refer to Table 4). When combined with river water (negligible $\mathrm{NaCl}$ concentration), the power output per unit flow of feed solution could be 3.3 MW. Using river water as the conjugated solution, however, would depend on the availability of the resource in the environment. For the Australian CSG sites, in particular, it is unlikely that river water could be used to feed a power plant, due to the scarcity of fresh water in these areas.

Another alternative for osmotic power production in Australia lies in the groundwater extracted by the gold mining industry. This industry heavily relies on the use of groundwater, in which 
the $\mathrm{NaCl}$ concentration varies from $1.5 \%$ to $20 \%$. This water is commonly disposed of in evaporation ponds, where even more concentrated solutions could be generated. The brine could be used to run an osmotic power plant. Examples of sites in Australia include the Pilbara and the Goldfields-Esperance regions, in Western Australia. It is estimated that the current licensed allocation of groundwater for the Northern Goldfields is about $90 \mathrm{hm}^{3} \mathrm{yr}^{-1}$ [62]. This is supplied wholly from local groundwater with $\mathrm{NaCl}$ concentration of up to $20 \%$. When paired with a fresh water solution (negligible salt concentration) or even brackish solution (salt concentration $\approx 1 \%$ ), the power output could be around 8 MW per unit flow of feed solution (refer to Table 4).

4.4. Sea water vs. brine from SWRO desalination plants (PRO-assisted desalination plant or SWRO-PRO hybrid system)

Feed solution: sea water

Draw solution: brine from SWRO desalination

Seawater Reverse Osmosis (SWRO) and Pressure Retarded Osmosis (PRO) hybrid systems (referred to as SWRO-PRO hybrid system or PRO-assisted desalination plants) consist of the symbiotic relationship between desalination and osmotic power processes [63-66]. Under these schemes, power is generated in a PRO plant attached to a desalination plant (Figure 7). This power is used in the desalination process. The PRO plant, in turn, uses the reject brine from the desalination process as draw solution and sea water as the feed solution. These hybrid systems are promising alternatives for the worldwide desalination industry, as they not only provide power to the desalination process, but also an opportunity to minimise the environmental impacts caused by the discharge of concentrated brine in the sea.

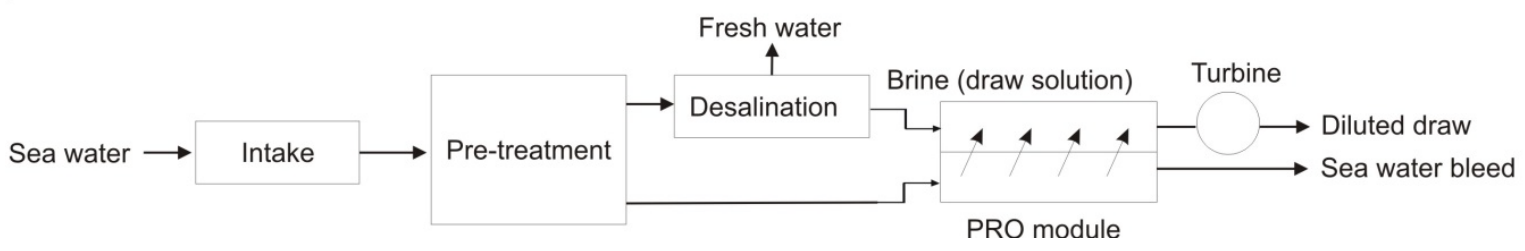

Figure 7. A conceptual scheme for a SWRO-PRO hybrid system. This scheme utilises reject brine from desalination plants (draw) and sea water (feed)

The power production from a SWRO-PRO hybrid system could be approximately 1.1 MW per $\mathrm{m}^{3} \mathrm{~s}^{-1}$ of feed solution (sea water) (refer to Table 4). The main advantage of this scheme is that sea water can be used as feed solution, which is plentiful and free. Additionally, the desalination 
plant could reduce its reliance on fossil fuels, contributing to reduced gas emissions to the atmosphere. Another advantage is that membranes for this combination of solutions are already being investigated for this purpose. In Table 3 it can be seen that there are many studies about membrane performance under PRO conditions using brine with $6 \% \mathrm{NaCl}$ concentration as draw solution and less concentrated solutions as feed. Commercially available membranes have been able to produce up to $5 \mathrm{~W} \mathrm{~m}^{-2}$ under an osmotic pressure differential similar to desalination brine and pure water [15]. More recently, customised membranes have been investigated, and power densities of $15.2 \mathrm{~W} \mathrm{~m}^{-2}$ [22], $20.9 \mathrm{~W} \mathrm{~m}^{-2}$ [25] and $24.3 \mathrm{~W} \mathrm{~m}^{-2}$ [21] have been observed under laboratory conditions for solutions simulating desalination brine and pure water.

The main disadvantage would probably be the high capital cost for the installation of membrane modules designed uniquely for power production and conversion of conventional SWRO plants to SWRO-PRO plants.

The Gold Coast Desalination Plant, in Queensland, Australia, can be used again as an example for the calculation of the osmotic power that could be produced under a realistic SWRO-PRO system. The Gold Coast plant requires 15 GWh per year of energy to generate 50,000 $\mathrm{m}^{3}$ day 1 of fresh water during a three-month period of operation. The plant, therefore, requires an input of 6.9 MW during the period of freshwater production. Assuming 40\% recovery [23], it can be estimated that the plant produces around $75,000 \mathrm{~m}^{3}$ day $^{-1}$ of concentrated brine during the operational period. This amount of brine can draw approximately the same volume (i.e., 75,000 $\mathrm{m}^{3}$ day $^{-1}$, or $0.9 \mathrm{~m}^{3} \mathrm{~s}^{-1}$ ) of sea water (feed solution). The mixing of these two solutions could generate about 1.0 MW (2.2 GWh over three months), if a power output of 1.1 MW per m $\mathrm{s}^{-1}$ of feed solution is assumed (refer to Table 4). This corresponds to $14.5 \%$ of the energy required in the desalination process. For the Gold Coast Desalination Plant, in particular, during the remaining nine months of the year, when the plant is inactive for freshwater production, the PRO plant could remain operational for power production. During this time, instead of using brine and sea water as draw and feed solutions, respectively, the plant would utilise river water (feed solution) and sea water (draw solution). As discussed in section 5.1.1, a power production of 2.8 MW would be possible with the available local water resources. This translates into an amount of energy of $18 \mathrm{GWh}$ over nine months. Together with the $2.2 \mathrm{GWh}$ generated from the brine, the total energy (20.2 GWh) is significantly higher than the energy required for the desalination process. 


\subsection{Waste water vs. sea water}

Feed solution: waste water

Draw solution: sea water

This PRO scheme is based on the utilisation of treated waste water as feed solution and sea water as draw solution, as illustrated in Figure 8. This scheme is another type of symbiotic relationship, in which the waste water treatment plant provides the feed solution to the power plant, and the power plant provides energy to the treatment plant.

The salinity of fluid waste streams from waste water treatment plants is typically as low as the salinity of river water. The salt concentration in waste water ranges from $0.03 \%$ to $0.5 \%$ [67]. This translates into an osmotic pressure differential of around 28 bar, and a power output of 1.4 MW per unit flow of waste water.

Regarding membranes for this particular scheme, customised thin-film composite membranes have already been tested using waste water as feed solution and sea water as draw solution by Chou et al. [18]. A power output of $4.1 \mathrm{~W} \mathrm{~m}^{-2}$ was achieved for a waste water solution with an $\mathrm{NaCl}$ concentration of $0.5 \%$, and a power density of $5.6 \mathrm{~W} \mathrm{~m}^{-2}$ was achieved for a waste water solution with an $\mathrm{NaCl}$ concentration of $0.2 \%$. These power densities are close to the breakeven point of $5 \mathrm{~W} \mathrm{~m}^{-2}$.

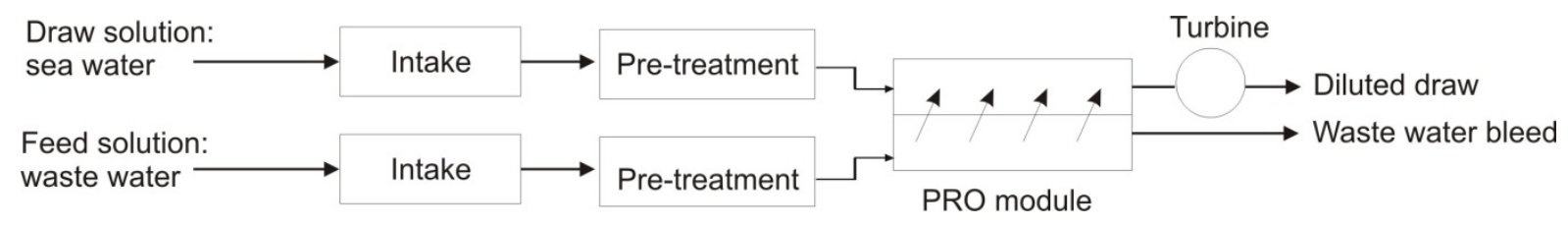

Figure 8. A conceptual scheme for a PRO plant using waste water (feed) and sea water (draw)

The city of Brisbane in Queensland, Australia, has a population of 2,146,577 which is projected to increase $17 \%$ by 2026 and $47 \%$ by 2051 [68]. Approximately $50 \%$ of the domestic and industrial effluents produced in Brisbane are treated in the Luggage Point Waste Water Treatment Plant (LPWWTP), located near the mouth of the Brisbane River, in Moreton Bay. It is estimated that the current discharge of the LPWWTP is $136,000 \mathrm{~m}^{3}$ day $^{-1}\left(1.6 \mathrm{~m}^{3} \mathrm{~s}^{-1}\right)$, and that this discharge will grow to $178,000 \mathrm{~m}^{3}$ day $^{-1}\left(2.1 \mathrm{~m}^{3} \mathrm{~s}^{-1}\right)$ by 2051 [31], which leads to 
power generation of around 2.3 MW and 3.0 MW, respectively. One option to increase the power production would be complementing the feed solution with fresh water from the Brisbane River. This river can securely provide $2 \mathrm{~m}^{3} \mathrm{~s}^{-1}$ of fresh water, as discussed in section 5.1.

4.6. Waste water vs. brine from SWRO desalination plants (SWRO-PRO-WWT hybrid system)

Feed solution: waste water

Draw solution: concentrated brine from SWRO desalination

Another symbiotic relationship using PRO is a hybrid scheme involving sea water reverse osmosis desalination (SWRO), osmotic power and waste water treatment (WWT). Under this scheme, the desalination plant provides the draw solution, while the waste water treatment plant provides the feed solution to the PRO plant. PRO energy is generated and used in either of the two other processes. This SWRO-PRO-WWT hybrid system has already been suggested and discussed in the literature (e.g., [23, 69, 70]) for a potential application of PRO. A conceptual ideal for this system is shown in Figure 9.

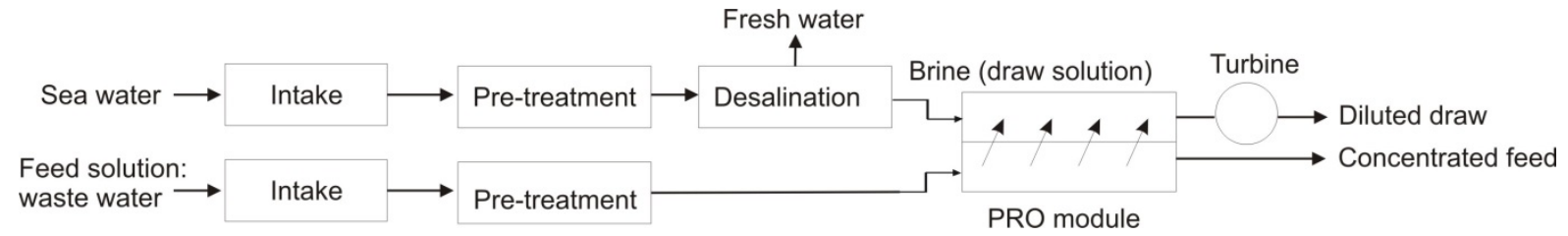

Figure 9. A conceptual scheme for a SWRO-PRO-WWT hybrid process. The draw and feed solutions are reject brine from desalination plants and effluent from waste water treatment plants, respectively

It is estimated that approximately 2.4 MW of power could be generated for each $\mathrm{m}^{3} \mathrm{~s}^{-1}$ of treated sewage using desalination brine as draw solution (refer to Table 4). In Brisbane, Australia, there is a project for the construction of a new desalination plant on the Fisherman's Island (located at the Brisbane River mouth in Moreton Bay, about 500 metres off the mainland [71]. On the opposite shore (continent side), lies the Luggage Point Waste Water Treatment Plant (LPWWTP). It is predicted that, when in operation, the new desalination plant on the Fisherman's Island will generate $3.3 \mathrm{~m}^{3} \mathrm{~s}^{-1}$ of brine (reject) [71]. As discussed in the previous section, the current discharge of the LPWWTP is $1.6 \mathrm{~m}^{3} \mathrm{~s}^{-1}$, and the prediction is $2.1 \mathrm{~m}^{3} \mathrm{~s}^{-1}$ by 2051 [31]. These flows have the potential to generate 3.8 MW and 5.0 MW, respectively, in a SWRO-PRO-WWT hybrid system. It should be noted that there is also the possibility of 
complementing the feed solution with water from the Brisbane River, and consequently, increase the power output.

An SWRO-PRO-WWT hybrid scheme would be a possible solution to the environmental problems caused by the desalination process (i.e., the direct disposal of brine into the sea), the by-products of the sewage treatment facilities and the high power demands of the desalination process. From the point of view of implementation, except for the PRO membranes, an SWROPRO-WWT hybrid scheme would require a small number of additional components, which are similar to those already standards in desalination industry.

Table 6. Main advantages and disadvantages of running an osmotic power plant with different combinations of solutions.

(Table 6 here - on a separate file)

\section{Main Technical Challenges for the Implementation of PRO in Large Scale}

With the currently available membrane technology, the capital costs associated with an osmotic power plant remain very high, making osmotic energy uncompetitive compared with other sources of renewable energy such as solar and wind [4]. The main components of the capital costs of an osmotic power plant are the hydro turbines, pumping and filtering systems, pressure exchangers and, most importantly, the membrane modules [4]. Due to the high susceptibility of the commercially-available membranes to concentration polarization, only marginal power densities have been able to be achieved. These are reported to vary between 0.5 and $2.5 \mathrm{~W} \mathrm{~m}^{-}$ 2 [4]. To increase power density, membranes that allow for higher water flux and salt rejection, with minimal internal concentration polarization and higher mechanical strength to support high hydraulic pressures have to be developed [72].

As a result of the low power density of the current membranes, the installed membrane area in a large scale osmotic power plant would have to be extremely large, reflecting directly on the capital costs of the plant. Since 2009 however, after the opening of the first prototype, the focus of PRO research has been primarily on developing new membranes for specific PRO application, aiming to obtain higher power outputs. Adepts of PRO believe the technology will become available "off-the-shelf” in about 5 to 10 years [48]. For PRO systems operating on fresh water (e.g., rivers) and sea water, the target has been to produce a membrane that is able 
to generate a minimum of $5 \mathrm{~W} \mathrm{~m}^{-2}$. Although this minimum power density has already been reached under laboratory conditions, the technology has not yet been proved to work in larger scales, in which concentration polarization is difficult to minimise. Also, issues such as membrane fouling and membrane resistance to the operating pressure are yet to be solved before PRO can be implemented in large-scale osmotic power plants. Recent figures show that the commercially-available thin-film composite membranes used at the Statkraft's osmotic power plant prototype have been producing a marginal power of $1 \mathrm{~W} \mathrm{~m}^{-2}$ [73]. However, even more recently, membrane developer Nitto Denko/Hydranautics signed an agreement with Statkraft to develop and supply a membrane that is designed specifically for PRO [74]. This membrane is characterized by high water permeability and low susceptibility to concentration polarization as a result of a reduced thickness, higher porosity and reduced tortuosity of the support layer as compared with commercial RO composite polyamide membranes [75]. This appears to be an important step towards upgrading PRO from laboratory and prototype scales to a commercial large-scale plant [4], though no results have been published on the advances made in this new membrane's development.

The issues of concentration polarization and membrane resistance are greater when it comes to draw solutions with concentrations higher than the concentration of sea water, such as concentrated brine from salt lakes or the reject brine from desalination plants. Research on membranes for this particular condition is also underway. The main issues to overcome in this case are the significantly higher osmotic pressure differences that the membranes will have to withstand, and the higher susceptibility to concentration polarization. Laboratory studies have found commercial membranes that could produce up to $60 \mathrm{~W} \mathrm{~m}^{-2}$ using pure water as feed solution and $18 \% \mathrm{NaCl}$ brine as draw solution [20].

There is also significant progress to be made regarding the development of suitable membrane modules. The standard spiral wound module designs have severe limitations regarding the internal flow pattern and pressure losses. Also, due to their low membrane area, the current modules are not adequate for scaling up to larger units. Given that an osmotic power plant would require millions of square meters of membrane, the PRO membrane modules should be equipped with at least several hundreds or even thousands of square meters of membrane [2, $4,76,77]$. Several design criteria have already been set for the design of membrane elements made specifically for PRO. These criteria include that the elements should: be able to convey 
flow on both sides of the membrane; contain a much larger membrane area; and be much more resistant to membrane fouling compared with the currently available membrane modules [2].

Fouling is also an important issue affecting membrane productivity and membrane life time, and thus costs, in PRO. Fouling can be partially reduced with pre-treatment of incoming solutions with physical separation processes, such as filtration. It should be noted that the energy applied in the pre-treatment process incurs reduction in net power, increasing the energy costs of the PRO plant. It has been suggested in the literature that groundwater, rather than river water, could be employed as feed solution as this water would be naturally filtered through the subsurface, reducing energy consumption in the pre-treatment process, and consequently risks of membrane fouling [1]. Intermittent osmotic backwashing of fouled membranes could be another way of reducing membrane fouling and keeping membrane productivity, as this would require only nominal pumping energy and supposedly pose negligible operational disruption [78]. Nevertheless, it is sensible to say that fouling is an issue that should be preferably addressed in membrane development (by developing fouling-resistant membranes, for instance) rather than in the development of pre-treatment and cleaning technologies.

Barriers to cost-effective energy production also include the minimization of parasitic process energy requirements associated with water conveyance and pre-filtration, which affect significantly the net power output of PRO systems.

\section{Final considerations}

Various methods for exploring salinity gradient energy using different combinations of solutions have been discussed in this article. Although the majority of the examples generally focus on Australian water resources, these PRO applications can also be projected to other locations in the world with similar conditions. This study has demonstrated that Australia, in particular, has a great potential for osmotic power production, with numerous favourable factors:

- The country's population continues to grow, increasing the demand for energy.

- The Australian Government has been funding research and development on new sources of renewable energy as a result of targets to reduce carbon emissions and to increase the supply of energy by renewable sources. 
- From the point of view of implementation of PRO, Australia has various sources of saline solutions that could be used as draw solutions in PRO plants. These include salt lakes, brine from desalination plants and saline groundwater.

- The largest Australian urban centres are located near the ocean and close to river mouths, an ideal condition for the construction of osmotic power plants.

- Growing demands for potable water have been driving momentum to construction of new desalination plants. These facilities are usually built as insurance, to guarantee the water supply during times of water scarcity. This means that these plants remain near idle for large periods of time, incurring high maintenance costs. If the same membrane modules could be applied in desalination and PRO processes, desalination plants could be designed to generate energy when they are not operating for freshwater production. This would significantly increase the utilisation efficiency of these facilities, and reduce ongoing costs. Alternatively, desalination plants working all year round could be partially powered by osmotic energy using the rejected brine from the desalination process as the draw solution in the power plant. The feed solution could be sea water, river water or even treated effluent from nearby wastewater treatment plants.

Although the technology behind osmotic power has been growing rapidly, osmotic power still remains several years away from commercial viability. The main issue to overcome regarding membranes is their susceptibility to concentration polarization (i.e., the gradual salt built-up on the membrane interfaces which considerably reduces the effective osmotic pressure difference between the feed and the draw solutions). Currently, the closest commercially available membranes for osmotic power are those produced for FO desalination, but their power density has been demonstrated to be low [47]. Membranes made specifically for osmotic power have not yet been produced on a commercial scale. However, Statkraft and Nitto Denko/Hydranautics have recently signed an agreement for the manufacturing of a specific membrane for PRO, which will hopefully be available "off-the-shelf" within a few years [79]. Nevertheless, while membranes for this particular purpose remain unavailable on a commercial scale, osmotic power will also remain commercially unviable.

There are other potential issues apart from the membrane technology. From the technical point of view, there is the issue of parasitic process energy requirements associated with water conveyance and pre-filtration, which significantly reduce the net power output. Also, the high 
susceptibility of membranes to fouling could significantly reduce the efficiency of a commercial power plant over time - an issue that has not been investigated at laboratory and prototype scales. Another potential issue is the difficulty that companies will face to obtain permits to build osmotic power plants, particularly in view of this being a new type of power technology. Therefore, it is necessary that the number of osmotic power plants prototypes be increased, so that these could be progressively scaled up to commercial units.

Therefore, several barriers must be overcome before PRO can be implemented on larger scales, as proposed in this study. Promisingly, however, each day research and development takes this technology forward. The progress made on PRO in the last few years is remarkable. There is a generalised belief that the right technology will become commercially available in less than five years. Additionally, this new form of renewable energy will be competitive with other renewable sources, but with the advantage of providing a steady power production and lower environmental impacts. The conceptual ideas presented in this article should be considered by all nations, particularly those with similar policies as Australia's, with incentives to foster the utilisation of renewable energy sources.

\section{Acknowledgements}

Funding for this project was provided by the Griffith Climate Change Response Program, the Griffith Sciences and the $\mathrm{CNPq}$ - Conselho Nacional de Desenvolvimento Científico e Tecnológico (Brazil).

\section{References}

[1] Yip NY, Elimelech M. Thermodynamic and energy efficiency analysis of power generation from natural salinity gradients by Pressure Retarded Osmosis. Environmental Science \& Technology. 2012;46:5230-9.

[2] Skilhagen SE. Osmotic power - a new, renewable energy source. Desalination and Water Treatment. 2010;15:271-8.

[3] Wick GL. Power from salinity gradients. Energy. 1978;3:95-100.

[4] Helfer F, Lemckert C, Anissimov YG. Osmotic power with Pressure Retarded Osmosis: Theory, performance and trends - a review. Journal of Membrane Science. 2014;453:337-58.

[5] Central Intelligence Agency. The World Factbook. Washington, DC: Central Intelligence Agency; 2011.

[6] Thorsen T, Holt T. The potential for power production from salinity gradients by pressure retarded osmosis. Journal of Membrane Science. 2009;335:103-10. 
[7] Fritzmann C, Löwenberg J, Wintgens T, Melin T. State-of-the-art of reverse osmosis desalination. Desalination. 2007;216:1-76.

[8] Loeb S. Osmotic power plants. Science. 1975;189:654-5.

[9] Loeb S. Production of energy from concentrated brines by pressure-retarded osmosis : I. Preliminary technical and economic correlations. Journal of Membrane Science. 1976;1:49-63.

[10] Loeb S, Van Hessen F, Shahaf D. Production of energy from concentrated brines by pressureretarded osmosis : II. Experimental results and projected energy costs. Journal of Membrane Science. 1976;1:249-69.

[11] Logan BE, Elimelech M. Membrane-based processes for sustainable power generation using water. Nature. 2012;488:313-9.

[12] Skilhagen SE, Dugstad JE, Aaberg RJ. Osmotic power - power production based on the osmotic pressure difference between waters with varying salt gradients. Desalination. 2008;220:476-82.

[13] Statkraft. Technology. 2012.

[14] Gerstandt K, Peinemann KV, Skilhagen SE, Thorsen T, Holt T. Membrane processes in energy supply for an osmotic power plant. Desalination. 2008;224:64-70.

[15] Achilli A, Cath TY, Childress AE. Power generation with pressure retarded osmosis: An experimental and theoretical investigation. Journal of Membrane Science. 2009;343:42-52.

[16] She $Q$, Jin $X$, Tang CY. Osmotic power production from salinity gradient resource by pressure retarded osmosis: Effects of operating conditions and reverse solute diffusion. Journal of Membrane Science. 2012;401-402:262-73.

[17] Schiestel T, Hänel C, Öxler L, Roelofs K, Walitza E. Celluloseacetate membranes with anoptimized internal structure for pressure retarded osmosis. 3rd Osmosis Membrane Summit. Barcelona2012.

[18] Chou S, Wang R, Shi L, She Q, Tang C, Fane AG. Thin-film composite hollow fiber membranes for pressure retarded osmosis (PRO) process with high power density. Journal of Membrane Science. 2012;389:25-33.

[19] Han G, Zhang S, Li X, Chung T-S. High performance thin film composite pressure retarded osmosis (PRO) membranes for renewable salinity-gradient energy generation. Journal of Membrane Science. 2013;440:108-21.

[20] Straub AP, Yip NY, Elimelech M. Raising the Bar: Increased Hydraulic Pressure Allows Unprecedented High Power Densities in Pressure-Retarded Osmosis. Environmental Science \& Technology Letters. 2013.

[21] Zhang S, Sukitpaneenit P, Chung T-S. Design of robust hollow fiber membranes with high power density for osmotic energy production. Chemical Engineering Journal (http://dxdoiorg/101016/jcej201310063).

[22] Song X, Liu Z, Sun DD. Energy recovery from concentrated seawater brine by thin-film nanofiber composite pressure retarded osmosis membranes with high power density. Energy \& Environmental Science. 2013;6:1199-210.

[23] Saito K, Irie M, Zaitsu S, Sakai H, Hayashi H, Tanioka A. Power generation with salinity gradient by pressure retarded osmosis using concentrated brine from SWRO system and treated sewage as pure water. Desalination and Water Treatment. 2012;41:114-21.

[24] Han G, Wang P, Chung T-S. Highly robust thin-film composite Pressure Retarded Osmosis (PRO) hollow fiber membranes with high power densities for renewable salinity-gradientenergygeneration. Environmental Science \& Technology. 2013;47:8070-7.

[25] Chou S, Wang R, Fane AG. Robust and high performance hollow fiber membranes for energy harvesting from salinity gradients by pressure retarded osmosis. Journal of Membrane Science. 2013;448:44-54.

[26] Syed A, Melanie J, Thorpe S, Penney K. Australian energy projections to 2029-30. Canberra:ABARE research report 10.02, prepared for the Department of Resources, Energy and Tourism; 2010.

[27] Climate Change Authority. Renewable Energy Target. Canberra: Australian Government; 2012.

[28] Clean Energy Future. An overview of the clean energy legislative package. Canberra: Australian Government; 2012. 
[29] Dopita M, Williamson R. Australia's renewable energy future. Canberra: Report for the Australian Academy of Science; 2010. p. 37.

[30] Kelada M. South Australia development - considering the osmotic power generationoption(white paper). Houston, Texas: MIK Technology; 2011. p. 12.

[31] CH2M HILL. Reuse of purified recycled water in South East Queensland. Brisbane: Report Rev 04, Reference 355570 prepared for the Queensland Water Commission; 2008. p. 71.

[32] Queensland Department of Natural Resources and Mines. Water Monitoring Data Portal. Queensland Department of Natural Resources and Mines, Brisbane; 2012.

[33] Department of the Environment Water Heritage and the Arts. Map of operating renewableenergy generators in Australia. Canberra2012.

[34] Crisp GJ. Energy Efficiency and Sustainability - Desalination Science. 1st Annual Desalination Conference. Irvine, CA2012.

[35] Hinkley J, Curtin B, Hayward J, Wonhas A, Boyd R, Grima C, et al. Concentrating solar power drivers and opportunities for cost-competitive electricity. CSIRO, Canberra; 2011. p. 27.

[36] International Renewable Energy Agency. Renewable Energy Technologies: Cost Analysis SeriesWind Power, Volume 1: Power Sector, Issue 5/5. International Renewable Energy Agency, Volume 1: PowerSector, Issue 5/5, Germany; 2012. p. 57.

[37] Zhu A, Christofides PD, Cohen Y. On RO membrane and energy costs and associated incentivesfor future enhancements of membrane permeability. Journal of Membrane Science. 2009;344:1-5.

[38] International Energy Agency. Energy technology perspectives: Scenarios and strategies to 2050. OECD/IEA, Paris; 2010.

[39] Hoang M, Bolto B, Haskard C, Barron O, Gray S, Leslie G. Desalination in Australia. Canberra: Report for the CSIRO Water for a Healthy Country National Research Flagship; 2009. p. 26.

[40] Sahin O, Siems R, Stewart RA, Porter M. Paradigm shift to enhanced water supply planning through augmented grids, scarcity pricing and adaptive factory water: A system approach. Environmental Modelling \& Software (Under review). 2014.

[41] Crisp GJ. Reducing carbon footprint of a seawater desalination plant - an Australian case study. MSSC Annual Salinity Summit Water and Energy. Las Vegas 2010.

[42] Collins KM. Desalination plant study to examine Tugun Desalination Plant operating options (Queensland Government media statement). Brisbane: The Queensland Cabinet and Ministerial Directory; 2012.

[43] Hydranautics. Membranes help to relieve water crisis in Australia. Membrane Technology. 2007;2007:10-1.

[44] Seqwater. Gold Coast Desalination Plant Tour. Gold Coast: Seqwater; 2010. p. 17.

[45] Hydranautics. Membranes help to relieve water crisis in Australia. Membrane Technology. 2007.

[46] Seqwater. Hinze Dam Stage 3 Project: Environmental Impact Statement. Gold Coast: Seqwater; 2007.

[47] Kho J. Osmotic power: A primer. San Francisco: Kachan \& Co; 2010. p. 18.

[48] Research and Markets. Analysing Osmotic Power. Dublin2012. p. 150.

[49] Kelada M. Global potential of hypersalinity osmotic power (white paper). Houston, Texas: MIK Technology; 2010. p. 12.

[50] Loeb S. One hundred and thirty benign and renewable megawatts from Great Salt Lake? Desalination. 2001;141:49-63.

[51] Kelada M. The Great Salt Lake osmotic power potential (white paper). Houston, Texas: MIK Technology; 2010. p. 7.

[52] Kelada M. Tunisia's current electrical energy tripled by hypersalinity osmotic power generation (white paper). Houston, Texas: MIK Technology; 2010. p. 12.

[53] Loeb S. Energy production at the Dead Sea by Pressure-Retarded Osmosis: Challenge or chimera? Desalination. 1998;120:247-62.

[54] Queensland Department of Natural Resources. Water Allocation and Management Planning (booklet). Queensland. Dept. of Natural Resources, Brisbane; 1997. p. 16. 
[55] National Land and Environment Audit. Water Resources in Australia - Surface and groundwateravailability and quality. Canberra: National Land and Water Resources Audit; 2001. p. 12.

[56] Economics Consulting Services. Water and the Western Australian Minerals and Energy Industry: Certainty of Supply for Future Growth. Perth: Report prepared for The Chamber of Minerals and Energy of Western Australia; 2004. p. 57.

[57] Rutovitz J, Harris S, Kuruppu N, Dunstan C. Drilling down - coal seam gas: A background paper. Sydney: Report prepared for the city of Sidney Council; 2011. p. 80.

[58] Biggs A, Witheyman S, Williams K, Cupples N, de Voil C, Power R, et al. Assessing the salinity impacts of coal seam gas water on landscapes and surface streams. Toowoomba: Final report of Activity 3 of the Healthy HeadWaters Coal Seam Gas Water Feasibility Study. Department of Natural Resources and Mines; 2012.

[59] Department of Employment, Economic Development and Innovation. Queensland's coal seam gas overview. Brisbane: Department of Employment, Economic Development and Innovation; 2011. p. 6.

[60] Parsons Brinckerhoff. Coal Seam Gas Water Management Study (Final Report). Brisbane: Report NRO0011 for the Department of Natural Resources, Mines and Energy; 2004. p. 105.

[61] Foster M, Matthew P. Arrow Energy Surat Gas Project, Agricultural Report, Surat Basin, Queensland. Robina, Australia: Technical report prepared for Coffey Environments PTY LTD; 2011.

[62] S. L. Johnson, Commander DP, O'Boy CA. Groundwater Resources of the Northern Goldfields, Western Australia. Hydrogeological Report Series. Perth: Report prepared for the Water and Rivers Commission; 1999. p. 57.

[63] Achilli A, Prante JL, Childress AE. The energetics of PRO-assisted desalination. 3rd Osmosis Membrane Summit. Barcelona2012.

[64] Kim J, Park M, Kim JH. Feasibility analysis of hybrid seawater reverse osmosis (SWRO) and pressure retarded osmosis (PRO) system. 3rd Osmosis Membrane Summit. Barcelona2012.

[65] Sim VST, She Q, Chong TH, Tang CY, Fane AG, Krantz WB. Strategicco-location in a hybrid process involving desalination and Pressure Retarded Osmosis (PRO). Membranes. 2013;3:98-125.

[66] Palacin LG, Tadeo F, Prada Cd, Touati K. Evaluation of the recovery of osmotic energy in desalination plants by using pressure retarded osmosis. Desalination and Water Treatment. 2012;51:360-5.

[67] Patterson RA. On-site treatment and disposal of septictank effluent. Armidale: UniversityofNew England; 1994.

[68] MWH. Regional Water Needs and Integrated Urban Water Management Opportunities. Brisbane: Report prepared for the Council of Mayors, South East Queensland, the Queensland Governmentand the Queensland Water Comission. ; 2007.

[69] Kim J, Lee J, Kim JH. Overview of pressure-retarded osmosis (PRO) process and hybrid application to sea water reverse osmosis process. Desalination and Water Treatment. 2012;43:193-200.

[70] Chung T-S, Li X, Ong RC, Ge Q, Wang H, Han G. Emerging forward osmosis (FO) technologies and challenges ahead for clean water and clean energy applications. Current Opinion in Chemical Engineering. 2012;1:246-57.

[71] SEQ Healthy Waterways Partnership. SEQ Regional Desalination Plant Siting Studies. Brisbane: Synthesis report prepared for the Queensland Water Commission; 2008. p. 18.

[72] Cath TY, Childress AE, Elimelech M. Forward osmosis: Principles, applications, and recent developments. Journal of Membrane Science. 2006;281:70-87.

[73] Statkraft. Result of Operation 2010 - 2012. 2012.

[74] Nitto Denko/Hydranautics. Statkraft and Nitto Denko/Hydranautics cooperate to make osmotic power a reality. http://www.membranes.com/press/STATKRAFT\%20NITTO\%20DENKO\%20HYDRANAUTICS\%2OPR\%2 06\%202011.pdf; 2011.

[75] HIROSE M. New Membrane for Osmotic Power. 3rd Osmosis Membrane Summit. Barcelona2012. 
[76] Skilhagen SE. Osmotic power: a new, renewable source of energy. 3rd Annual European Renewable Energy Markets. Berlin2012.

[77] Kleverud J, Skilhagen SE, Brekke G. Experiences with the Tofte prototype plant. 3rd Osmosis Membrane Summit. Barcelona2012.

[78] Yip NY, Elimelech M. Influence of Natural Organic Matter Fouling and Osmotic Backwash on Pressure Retarded Osmosis Energy Production from Natural Salinity Gradients. Environmental Science \& Technology. 2013.

[79] Hal per M. Osmotic power pushes closer to reality. Smartplanet: CBS Interactive; 2011. 